\title{
Dinoflagellate resting cysts from surface sediments of the Adriatic Ports: Distribution and potential spreading patterns
}

\author{
Elena Di Poi ${ }^{\mathrm{a}, 1}$, Romina Kraus ${ }^{\mathrm{b}, *, 1}$, Marina Cabrini ${ }^{\mathrm{a}}$, Stefania Finotto ${ }^{\mathrm{c}}$, Vesna Flander-Putrle ${ }^{\mathrm{d}}$, \\ Mateja Grego ${ }^{\mathrm{d}}$, Nataša Kužat ${ }^{\mathrm{b}}$, Živana Ninčević Gladan ${ }^{\mathrm{e}}$, Laura Pezzolesi ${ }^{\mathrm{f}}$, Elena Riccardi ${ }^{\mathrm{g}}$, \\ Fabrizio Bernardi Aubryc, Mauro Bastianini ${ }^{c}$ \\ a Istituto Nazionale di Oceanografia e di Geofisica Sperimentale (OGS), Section of Oceanography, Via A. Piccard 54, 34151 Trieste, Italy \\ ${ }^{\mathrm{b}}$ Ruđer Bošković Institute, Center for Marine Research, Giordano Paliaga 5, 52210 Rovinj, Croatia \\ ${ }^{\mathrm{c}}$ ISMAR CNR Institute of Marine Sciences, National Research Council of Italy, Castello 2737/f, 30122 Venice, Italy \\ ${ }^{\mathrm{d}}$ National Institute of Biology, Marine Biology Station, Fornače 41, 6330 Piran, Slovenia \\ e Institute of Oceanography and Fisheries, Šetalište I. Meštrovića 63, 21000 Split, Croatia \\ ${ }^{\mathrm{f}}$ Department of Biological, Geological and Environmental Sciences (BiGeA), University of Bologna, Via Sant'Alberto 163, 48123 Ravenna, Italy \\ ${ }^{\mathrm{g}}$ Fondazione Centro Ricerche Marine, National Reference Laboratory on Marine Biotoxins, V.le A. Vespucci 2, 47042 Cesenatico, FC, Italy
}

\section{A R T I C L E I N F O}

\section{Keywords:}

Dinoflagellate cysts

Non-indigenous species

Port baseline survey

Ballast waters

Circulation

Adriatic Sea

\begin{abstract}
A B S T R A C T
The ability of microalgae to preserve viable in coastal sediments as resting forms provides a reservoir of biodiversity and a useful tool to determine species spreadings. This study represents the first port baseline survey on dinoflagellate cysts, investigated in nine Adriatic ports during a cross border project. 40 dinoflagellate taxa were detected. The assemblages resulted in all ports dominated by Lingulodinium polyedra and Alexandrium minutum/ affine/tamutum group. General separation to the western and eastern side of the Adriatic regarding cysts assemblage composition, partially abundance, was observed. Six taxa were detected as non-indigenous species for the Adriatic. Two taxa are included in the list of harmful aquatic organisms, indicating the potential threat of ballast waters in the Adriatic. Potential spreading of taxa by general circulation and ballast waters, intra- and extra-Adriatic was investigated. The entering in to force of the ballast waters management regulations should enhance prospects to minimize future harmful impacts.
\end{abstract}

\section{Introduction}

Coastal waters host many planktonic organisms that spend most of their lifecycle in a resting phase (Boero et al., 1996). Benthic cysts play a pivotal role in the ecology of dinoflagellates (Walker, 1984; Dale, 1983), since they allow survival through adverse environmental conditions, such as nutrient depletion, grazing, competition, high turbulence and temperature decrease (Figueroa and Bravo, 2005; Nagai et al., 2002; Pospelova et al., 2008). Cyst-forming species are not equally distributed among the extant dinoflagellate orders, being much more common for the Peridiniales, Gonyaulacales and Gymnodiniales, and quite rare for the Dinophysiales and Prorocentrales (Faust, 1990, 1993; Moita and Sampayo, 1993; Head, 1996). Cells are not-motile and are commonly produced through sexual reproduction (Walker, 1984). Cysts are surrounded by a dense, resistant wall, which cause their settling on the sea bottom (Montresor et al., 1998). Their ability to preserve viable in sediments for decades provides a reservoir of biodiversity and a suitable tool to study temporal changes in phytoplankton populations in a given area (Lundholm et al., 2011). Cysts also act as "seeds" for initiation of blooms once favourable conditions are re-established (Pfiester and Anderson, 1987). In the phylum Dinophyta, at least 200 species can produce cysts (Dale and Dale, 2002), and more than 16 species are known to be toxic and cause harmful red-tide events (Matsuoka and Fukuyo, 2000). This number is expected to increase even further since new cyst morphotypes continue to be discovered (Satta et al., 2010). Surveys of cyst assemblages in recent sediments are

\footnotetext{
* Corresponding author.

E-mail addresses: edipoi@inogs.it (E. Di Poi), kraus@cim.irb.hr (R. Kraus), mcabrini@inogs.it (M. Cabrini), stefania.finotto@ve.ismar.cnr.it (S. Finotto), flander@nib.si (V. Flander-Putrle), mateja.grego@nib.si (M. Grego), nkuzat@irb.hr (N. Kužat), nincevic@izor.hr (Ž. Ninčević Gladan), laura.pezzolesi@unibo.it (L. Pezzolesi), riccardi.elena11@gmail.com (E. Riccardi), fabrizio.bernardi@ismar.cnr.it (F. Bernardi Aubry), mauro.bastianini@ismar.cnr.it (M. Bastianini).

${ }^{1}$ These authors contributed equally to the work.
} 
thus important to forecasting the occurrence of future bloom events and give early warnings of the presence of toxic or non-toxic species (Anderson et al., 1983, 2003; Giannakourou et al., 2005; Lundholm et al., 2011). Information on the distribution and abundance of cysts is also essential for understanding the ecology and bloom dynamic of many harmful aquatic organisms (HAOs) (Joyce, 2004; Rubino et al., 2010, 2017). The application of cyst assemblage studies to harmful algal species will extend knowledge of these microalgae; this approach increases the likelihood of detecting the occurrence of species not previously reported from particular areas (e.g., Alexandrium species; Montresor et al., 1998; Joyce et al., 2005; Bravo et al., 2006). Cysts distribution dynamics could be controlled by various biological and environmental factors: primary production and physico-chemical conditions such as sea-surface temperature, sea-surface salinity (Candel et al., 2012), including sediment physical characteristics (Anderson et al., 2005; Anglès et al., 2010; Horner et al., 2011). Several studies suggest that highest cyst abundances are correlated with fine sediment fractions (Triki et al., 2017).

The Adriatic is located in the northernmost part of the Mediterranean. The economic development and social existence of their countries depend on the preservation of the basin, which is an important international and local seaway subjected to intense commercial maritime traffic. For these reasons, Adriatic ports, also featured by shallow water, low hydrodynamic forcing, can represent a reservoir of biodiversity and potential hotspots of species dispersions (Dale, 1983; Belmonte et al., 1995; Garcés et al., 1999). In such areas, and especially those hosting shellfish farms, where the transfer of organisms occurs intentionally, the risk of introducing Non-Indigenous Species (NIS) can be high (Hégaret et al., 2008). Biological invasions can also happen unintentionally, with the maritime traffic.

The International Maritime Organization (IMO) has adopted in 2004 the "International Convention for the Control and Management of Ship's Ballast Water and Sediments" (BWM Convention), aimed to reduce the spread of Harmful Aquatic Organisms and Pathogens (HAOP) among ports and areas, by establishing standards and procedures for the management and control of ships' ballast waters (BW) and sediments (Cabrini et al., 2019; this Issue). BW is seawater carried in ships' ballast tanks to improve stability, balance and trim. It is taken up or discharged when cargo is unloaded or loaded, or when a ship needs extra stability in foul weather. Ballast water discharge can introduce potential HAOP causing negative impact to aquatic ecosystems, human health and economic activities worldwide. BW studies conducted in different world's oceans, have demonstrated that ships are involved in the transfer of aquatic organisms across natural boundaries (Howarth, 1981; Carlton, 1985; Williams et al., 1988; Hallegraeff, 1993; Rigby and Hallegraeff, 1996; Macdonald and Davidson, 1997; Hay et al., 1997; Gollasch, 1998; Gollasch et al., 2000a, 2000b; Olenin et al., 2000; Ruiz et al., 2000; Wonham et al., 2001; Gollasch, 2002; Gollasch et al., 2002; Murphy et al., 2002; Coutts et al., 2003). It is estimated that BW are responsible for the transfer of approximately 7.000-10.000 different marine species globally every day (Carlton and Geller, 1993; Carlton, 1999; Gollasch, 2002). In the last decades, introductions have increased in frequency, volume, and in the degree of global biotic homogeneity (Lodge, 1993). In shipping, organisms are transferred inside ballast tanks, in the sediments and/or attached to the ship's hull. The introduction of NIS via ships' BW can influence marine ecosystems by causing major alterations in the composition and function of ecological communities' worldwide (Carlton, 1987, 1989; Lodge, 1993; Williamson, 1996). Not all the organisms survive in tanks, but some of them after the release establish populations in new locations. Some species have already harmfully impacted natural environments, human health and caused economic losses (David et al., 2007) by harming aquaculture farms by diminishing availability of healthy stocks (Graneli et al., 1998; Camacho et al., 2007; Hallegraeff, 2010). New invasions will intensify, since trades and transport routes are expanding to regions around the world (Carlton, 1985; Carlton, 1989; Chapman and
Carlton, 1991; Baldwin, 1992; Berman et al., 1992; Hutchings, 1992; Carlton and Geller, 1993; Buttermore et al., 1994). In intra-coastal voyages, with shorter resilience days in tanks, organisms have greater possibility to survive; indeed, several studies suggest that intra-coastal transport may be even more significant in the spread of organisms (Williams et al., 1988). BW are proven vector of spreading phytoplankton species, including dinoflagellate vegetative and resting stages, over the large distances across the oceans (Baldwin, 1992; Hallegraeff et al., 1990; Hamer et al., 2000). Indeed, high densities and wide diversities of dinoflagellate cysts are reported to withstand the severe conditions existent within ballast tanks, such as absence of light, reduced food availability and strong fluctuations of physico-chemical conditions (Hallegraeff and Bolch, 1991, 1992; Kelly, 1993; Chu et al., 1997; Macdonald and Davidson, 1998).

The aim of this study, conducted in the frame of the EU IPA Adriatic BALMAS project (Ballast water management system for Adriatic Sea protection), was to perform a Port Baseline Survey (PBS) in order to investigate on the species composition, abundance and distribution of dinoflagellate cyst assemblages in 9 Adriatic ports located along the Italian, Slovenian and Croatian coasts. A special effort was carried out on the detection of a baseline list of potentially HAOs and NIS, and on the tracking of the possible relationship between cyst presence and the BW transport. To date, no cross-border study evaluated both cyst diversity and abundance and the potential effect of harmful dinoflagellate cysts in the Adriatic port surface sediments.

\section{Methods}

\subsection{Study sites (characterization of the ports)}

Since major ports are often the first places where marine species are introduced, the aim of the sampling and laboratory activities, were to provide information about the presence and possible negative effects of both non-indigenous and native harmful species in ports, as hotspots of BW discharges. Therefore, an extensive field sampling performed twice a year (in spring and autumn when possible) was conducted in 9 Adriatic ports along the Italian (Trieste, Venice, Ancona, Bari), the Slovenian (Koper) and the Croatian (Pula, Rijeka, Šibenik, Split) coasts (Fig. 1, Table 1). Sampling was performed according to the BALMAS PBS procedures (Ninčević Gladan et al., 2014) based on the CRIMP protocol (Hewitt and Martin, 2001), modified in order to meet specific needs of the Adriatic Sea.

All selected ports are characterised by intense marine traffic and thus subjected to direct (ballast water-bw) or indirect (channel marker $\mathrm{chm}$ ) influence of BW, further in text: impacted (bw). Industrial areas, sites hosting or close to cargo, ferryboat and tanker terminals, with current/former shipping operations were selected for the purpose of this study. Adjacent sites outside ports, with presumably lower impact of BW, were contemporary sampled as control stations, including reference - ref., and anchorage - anc, further in text: control (ref). A complete list of the BALMAS PBS sampling sites with maps and coordinates are given in Kraus et al. (2019a). Short account of samples collection, applied treatments and analysis of samples is given in Table 2.

\subsection{Phytoplankton sampling and analysis}

Phytoplankton was sampled both for quantitative and qualitative analyses at each station and the frequency was designed to cover two seasons, spring/autumn. For detailed description of the sampling method and microscopic analyses of phytoplankton we refer to Mozetič et al. (this issue).

\subsection{Sediment sampling and processing}

Sampling of sediments was performed according to the BALMAS 


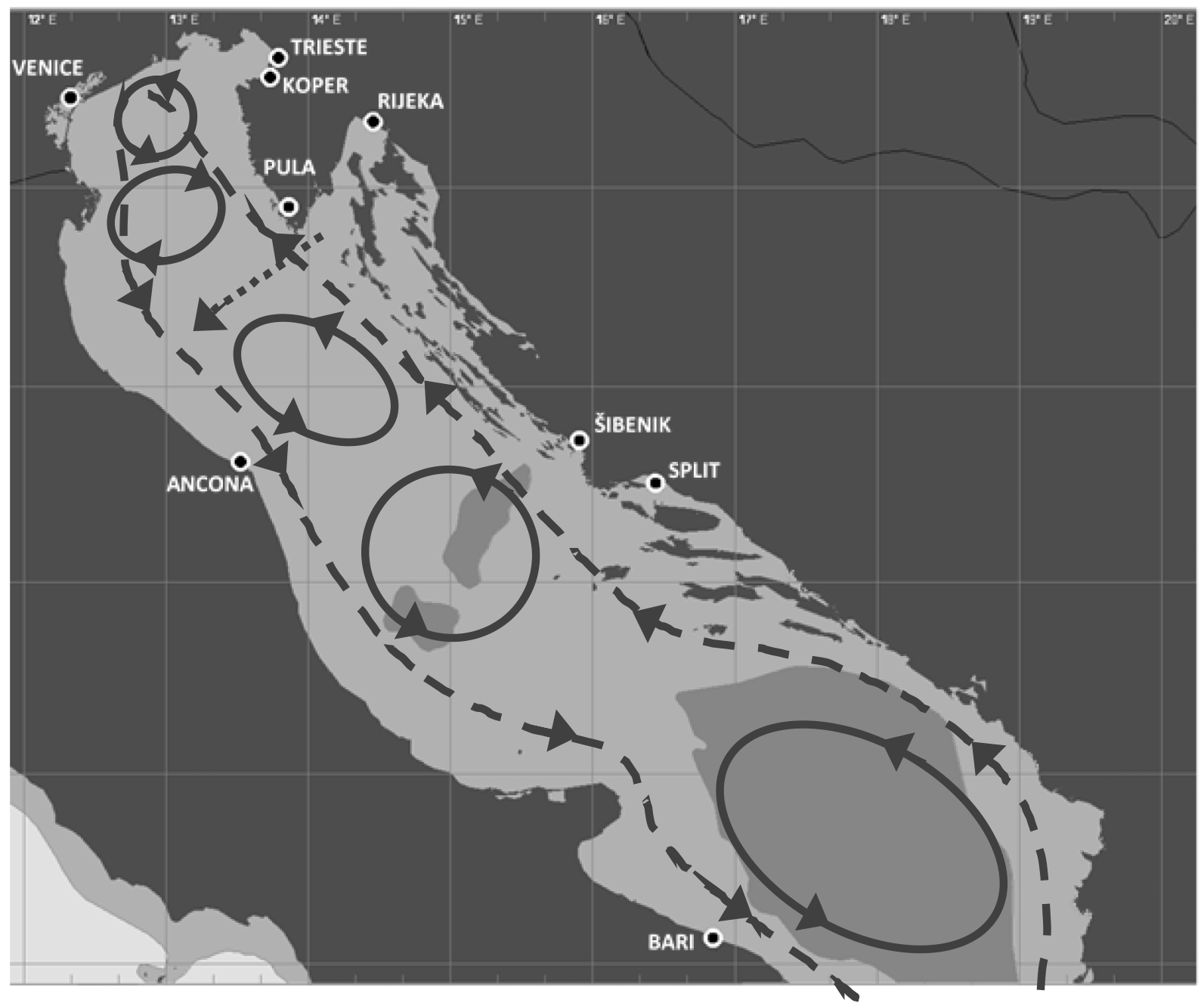

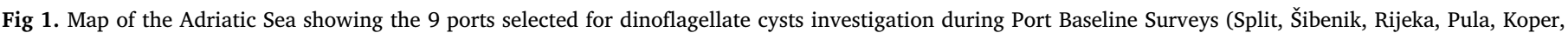

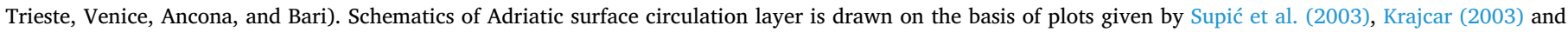
Kraus et al. (2019b); general circulation pattern (dashed line), gyres (solid) and current induced by Bora episodes (dotted).

guidelines, proposed in the PBS protocol (Kraus et al., 2019a), using either scuba divers or benthic cores and grabs, when the manual collection of sediments was not applicable. All samples were pre-treated and processed as described in Table 2. Cleaning and concentration of cysts from surface sediments was performed following the equivalent methodological procedures described in the PBS protocol according to Blanco (1986), Bolch (1997) or the palinological procedures as stated in Matsuoka and Fukuyo (2000) (Table 2). All samples were stored in the dark at $4{ }^{\circ} \mathrm{C}$ until microscopical analysis. Sub-samples were collected to obtain DW by drying the samples at $105^{\circ} \mathrm{C}$ for one week.

\subsection{Microscopic analysis}

Aliquots of final samples were counted in sedimentation chambers with inverted light microscopes at 200 and $400 \times$ magnifications, according to the Utermöhl (1958) method and Zingone et al. (2010) (Table 2). Counts were carried out noting the number of viable cysts of each species in half of sedimentation chamber. Dinoflagellate cysts were identified based on available morphological characteristics, such as the shape of cyst body and its ornamentation, wall structure and color, and types of aperture or archeopyle as stated in Matsuoka and Fukuyo (2000), Bringue et al. (2016), Kokinos and Anderson (1995), Pitcher and Joyce (2009), Rochon et al. (1999), Wang et al. (2007). Morphological identification was performed for cysts with distinctive species-specific morphological features. However, many cysts could not be identified at the species level mainly as available taxonomic keys do not always exist among related species, and/or available morphological features largely vary in the same species among different environments (Ellegaard, 2000; Matsuoka et al., 2009). Alternatively, the corresponding vegetative forms were used for species identification through germination of some cysts (Gu et al., 2013; Ellegaard et al., 2003). Unfortunately, the germination of many cysts could not be successfully achieved in each port since some of the laboratories were not equipped for germination experiments. Empty cysts were not considered for the identification. Densities were expressed in number of cysts per gram of dry sediment $\left(\mathrm{g}^{-1} \mathrm{DW}\right)$.

\subsection{Statistical analysis}

For statistical analyses of cysts dataset PRIMER (Plymouth Routines In Multivariate Ecological Research) v6 and PERMANOVA + add on to PRIMER software package was used (Clarke and Warwick, 2001). Routines applied were Cluster analysis, MDS, ANOSIM and SIMPER. As a transformation method, 4th root transformation was applied, to down-weight considerably the impact of the abundant taxa, allowing the rare taxa to exert some influence on the similarity calculations. For similarity measures Bray-Curtis coefficient was used. Cluster analysis was performed using group-average linking. 
Table 1

List of the BALMAS PBS sampling sites with depths and coordinates in nine Adriatic ports where dinoflagellate cysts were investigated. The name of each sampling site is a compound of: port name (in capital letters), type of sampling site (bw = directly impacted by ballast water, $\mathrm{chm}=$ channel marker site, anc $=$ anchorage site, and ref. = control site), and a suffix identifying the institution in charge of the PBS (internal reference coding, e.g. J1, K1, 4A...).

\begin{tabular}{|c|c|c|c|c|c|}
\hline Country & PORT & $\begin{array}{l}\text { Sampling } \\
\text { station }\end{array}$ & Depth & Latitude & Longitude \\
\hline \multirow[t]{14}{*}{ Croatia } & \multirow[t]{4}{*}{ Split } & STbwJ1 & 17.0 & $43^{\circ} 32^{\prime} 18.42^{\prime \prime} \mathrm{N}$ & $16^{\circ} 26^{\prime} 21.01^{\prime \prime} \mathrm{E}$ \\
\hline & & STbwK1 & 17.0 & $43^{\circ} 32^{\prime} 05.71^{\prime \prime} \mathrm{N}$ & $16^{\circ} 27^{\prime} 48.42^{\prime \prime} \mathrm{E}$ \\
\hline & & STbwL1 & 10.0 & $43^{\circ} 31^{\prime} 40.98^{\prime \prime} \mathrm{N}$ & $16^{\circ} 28^{\prime} 07.75^{\prime \prime} \mathrm{E}$ \\
\hline & & STbwL2 & 15.0 & $43^{\circ} 31^{\prime} 44.65^{\prime \prime} \mathrm{N}$ & $16^{\circ} 27^{\prime} 49.25^{\prime \prime E}$ \\
\hline & \multirow[t]{2}{*}{ Šibenik } & SIbw1 & 15.0 & $43^{\circ} 43^{\prime} 30.54^{\prime \prime} \mathrm{N}$ & $15^{\circ} 53^{\prime} 57.41^{\prime \prime} \mathrm{E}$ \\
\hline & & SIbw3 & 33.0 & $43^{\circ} 44^{\prime} 37.68^{\prime \prime N}$ & $15^{\circ} 52^{\prime} 50.77^{\prime \prime} \mathrm{E}$ \\
\hline & \multirow[t]{4}{*}{ Rijeka } & RIbwB & $7.0-8.8$ & $45^{\circ} 19^{\prime} 42.42^{\prime \prime} \mathrm{N}$ & $14^{\circ} 25^{\prime} 50.16^{\prime \prime} \mathrm{E}$ \\
\hline & & RIbwS & $7.3-8.2$ & $45^{\circ} 19^{\prime} 20.40^{\prime \prime} \mathrm{N}$ & $14^{\circ} 26^{\prime} 19.68^{\prime \prime} \mathrm{E}$ \\
\hline & & RIchm & $56.7-57.2$ & $45^{\circ} 19^{\prime} 30.30^{\prime \prime} \mathrm{N}$ & $14^{\circ} 23^{\prime} 49.38^{\prime \prime} \mathrm{E}$ \\
\hline & & RIref & $30.2-35.5$ & $45^{\circ} 18^{\prime} 51.06^{\prime \prime} \mathrm{N}$ & $14^{\circ} 28^{\prime} 08.82^{\prime \prime} \mathrm{E}$ \\
\hline & \multirow[t]{4}{*}{ Pula } & PUbwC & $7.0-7.8$ & $44^{\circ} 51^{\prime} 59.46^{\prime \prime} \mathrm{N}$ & $13^{\circ} 49^{\prime} 41.82^{\prime \prime} \mathrm{E}$ \\
\hline & & PUbwS & $5.7-6.2$ & $44^{\circ} 53^{\prime} 30.54^{\prime \prime} \mathrm{N}$ & $13^{\circ} 48^{\prime} 07.74^{\prime \prime} \mathrm{E}$ \\
\hline & & PUchm & $12.1-12.5$ & $44^{\circ} 52^{\prime} 43.20^{\prime \prime} \mathrm{N}$ & $13^{\circ} 50^{\prime} 24.00^{\prime \prime} \mathrm{E}$ \\
\hline & & PUref & $33.7-36.7$ & $44^{\circ} 52^{\prime} 33.78^{\prime \prime} \mathrm{N}$ & $13^{\circ} 47^{\prime} 34.74^{\prime \prime} \mathrm{E}$ \\
\hline \multirow[t]{4}{*}{ Slovenia } & \multirow[t]{4}{*}{ Koper } & KOanc4A & 17.0 & $45^{\circ} 33^{\prime} 48.66^{\prime \prime} \mathrm{N}$ & $13^{\circ} 42^{\prime} 52.86^{\prime \prime} \mathrm{E}$ \\
\hline & & KObw1A & 10.0 & $45^{\circ} 33^{\prime} 05.10^{\prime \prime} \mathrm{N}$ & $13^{\circ} 44^{\prime} 11.04^{\prime \prime} \mathrm{E}$ \\
\hline & & KObw2A & 13.1 & $45^{\circ} 33^{\prime} 27.36^{\prime \prime} \mathrm{N}$ & $13^{\circ} 44^{\prime} 12.00^{\prime \prime} \mathrm{E}$ \\
\hline & & KObw3A & 12.5 & $45^{\circ} 33^{\prime} 52.44^{\prime \prime} \mathrm{N}$ & $13^{\circ} 44^{\prime} 11.10^{\prime \prime} \mathrm{E}$ \\
\hline \multirow[t]{14}{*}{ Italy } & \multirow[t]{3}{*}{ Venice } & VEancPTF & 16.0 & $45^{\circ} 18^{\prime} 49.79^{\prime \prime} \mathrm{N}$ & $12^{\circ} 30^{\prime} 31.79^{\prime \prime} \mathrm{E}$ \\
\hline & & VEbwP1 & 12.0 & $45^{\circ} 26^{\prime} 13.17^{\prime \prime} \mathrm{N}$ & $12^{\circ} 18^{\prime} 39.98^{\prime \prime E}$ \\
\hline & & VEbwW2 & 7.0 & $45^{\circ} 26^{\prime} 54.19^{\prime \prime} \mathrm{N}$ & $12^{\circ} 15^{\prime} 29.87^{\prime \prime} \mathrm{E}$ \\
\hline & \multirow[t]{5}{*}{ Trieste } & TSbw1 & 18.5 & $45^{\circ} 38^{\prime} 01.08^{\prime \prime} \mathrm{N}$ & $13^{\circ} 45^{\prime} 09.24^{\prime \prime} \mathrm{E}$ \\
\hline & & TSbw2 & 16.0 & $45^{\circ} 37^{\prime} 47.22^{\prime \prime} \mathrm{N}$ & $13^{\circ} 46^{\prime} 10.98^{\prime \prime} \mathrm{E}$ \\
\hline & & TSbw3 & 14.0 & $45^{\circ} 37^{\prime} 04.08^{\prime \prime} \mathrm{N}$ & $13^{\circ} 46^{\prime} 30.96^{\prime \prime} \mathrm{E}$ \\
\hline & & TSbw4 & 14.0 & $45^{\circ} 36^{\prime} 41.04^{\prime \prime} \mathrm{N}$ & $13^{\circ} 46^{\prime} 30.96^{\prime \prime} \mathrm{E}$ \\
\hline & & TSrefC1 & 18.0 & $45^{\circ} 42^{\prime} 03.00^{\prime \prime} \mathrm{N}$ & $13^{\circ} 42^{\prime} 36.00^{\prime \prime} \mathrm{E}$ \\
\hline & \multirow[t]{3}{*}{ Ancona } & ANancAPI & $13.0-16.0$ & $43^{\circ} 40^{\prime} 29.72^{\prime \prime} \mathrm{N}$ & $13^{\circ} 24^{\prime} 34.37^{\prime \prime} \mathrm{E}$ \\
\hline & & ANbwDS & $10.0-11.0$ & $43^{\circ} 37^{\prime} 12.97^{\prime \prime N}$ & $13^{\circ} 29^{\prime} 48.20^{\prime \prime} \mathrm{E}$ \\
\hline & & ANbwLR & $5.0-6.0$ & $43^{\circ} 37^{\prime} 30.91^{\prime \prime N}$ & $13^{\circ} 29^{\prime} 40.00^{\prime \prime} \mathrm{E}$ \\
\hline & \multirow[t]{3}{*}{ Bari } & BIbw1 & $10.0-12.0$ & $41^{\circ} 08^{\prime} 19.89^{\prime \prime} \mathrm{N}$ & $16^{\circ} 51^{\prime} 57.30^{\prime \prime} \mathrm{E}$ \\
\hline & & BIbw2 & $4.5-7.5$ & $41^{\circ} 08^{\prime} 00.17^{\prime \prime N} \mathrm{~N}$ & $16^{\circ} 52^{\prime} 05.92^{\prime \prime} \mathrm{E}$ \\
\hline & & BIbw3 & $3.5-4.5$ & $41^{\circ} 08^{\prime} 20.59^{\prime} \mathrm{N}$ & $16^{\circ} 50^{\prime} 55.59^{\prime \prime} \mathrm{E}$ \\
\hline
\end{tabular}

\section{Results}

\subsection{Taxa and cyst densities}

Summarized information on abundance and total number of observed taxa is given in Table 3. Abundance ranged from minimum of 4 cysts $\mathrm{g}^{-1}$ DW (in port of Venice, at impacted stations in May and October 2014) to the maximal 11,554 cysts $\mathrm{g}^{-1}$ DW (in port of Split, in April 2011, due to high contribution of $L$. polyedra, contribution of $90 \%$ ). The highest number of observed taxa per single sample was observed in port of Trieste, 20 taxa per sample, followed by 15 in Koper and Split, and lowest, only three taxa in Venice and Ancona (Table 3). At port level, the highest diversity (number of observed taxa) was observed in port of Trieste (30 in the entire port, 29 taxa at impacted and 18 at control stations (Table 4). During sampling in March, 27 taxa was found at impacted and 15 at control stations, while in May, taxa count was lower, 21 and 12, respectively. The lowest diversity characterised Ancona and Venice, where spring/summer and autumn count, at both impacted and control stations, ranged from 3 to 5 . The lowest cysts diversity, only three observed taxa, was reported for impacted stations during May and July, in Venice and Ancona, respectively (Table 4).

\subsection{Cyst assemblages}

Complete taxa listing of identified cysts during PBS performed in nine ports is presented in Table 4.

Taxa observation is reported per specific port, month of sampling and type of sampling station. Samplings were performed at sampling
Table 2

Used devices and followed methods in dinoflagellate cysts sample collection, treatment and microscopic analyses in nine Adriatic ports.

\begin{tabular}{|c|c|c|c|c|c|c|c|}
\hline \multirow{2}{*}{ Port } & \multirow{2}{*}{ Month } & \multicolumn{3}{|c|}{ Observed taxa } & \multicolumn{3}{|c|}{ Cysts abundance $\left(\mathrm{g}^{-1} \mathrm{DW}\right)$} \\
\hline & & Ave & Min & Max & Ave & Min & Max \\
\hline \multirow{6}{*}{ SPLIT } & 4 & 12 & 9 & 15 & 3399 & 252 & 11554 \\
\hline & 6 & 7 & 7 & 7 & 680 & 680 & 680 \\
\hline & 10 & 8 & 8 & 8 & 1060 & 1060 & 1060 \\
\hline & ref & n.a. & n.a. & n.a. & n.a. & n.a. & n.a. \\
\hline & bw & n.a. & n.a. & n.a. & n.a. & n.a. & n.a. \\
\hline & in total & 10 & 7 & 15 & 2556 & 252 & 11554 \\
\hline \multirow{5}{*}{ ŠIBENIK } & 6 & 7 & 3 & 10 & 2745 & 610 & 4880 \\
\hline & 11 & 6 & 5 & 7 & 350 & 300 & 400 \\
\hline & ref & n.a. & n.a. & n.a. & n.a. & n.a. & n.a. \\
\hline & bw & n.a. & n.a. & n.a. & n.a. & n.a. & n.a. \\
\hline & in total & 6 & 3 & 10 & 1547 & 300 & 4880 \\
\hline \multirow{5}{*}{ RIJEKA } & 4 & 7 & 5 & 10 & 130 & 64 & 204 \\
\hline & 12 & 7 & 4 & 9 & 109 & 49 & 149 \\
\hline & ref & 7 & 7 & 7 & 89 & 64 & 113 \\
\hline & bw & 7 & 4 & 10 & 130 & 49 & 204 \\
\hline & in total & 7 & 4 & 10 & 120 & 49 & 204 \\
\hline \multirow{5}{*}{ PULA } & 5 & 8 & 6 & 10 & 328 & 80 & 516 \\
\hline & 12 & 7 & 5 & 10 & 330 & 44 & 763 \\
\hline & ref & 8 & 7 & 8 & 336 & 224 & 449 \\
\hline & bw & 7 & 5 & 10 & 326 & 44 & 763 \\
\hline & in total & 7 & 5 & 10 & 329 & 44 & 763 \\
\hline \multirow{5}{*}{ KOPER } & 6 & 13 & 10 & 15 & 588 & 372 & 805 \\
\hline & 11 & 12 & 10 & 14 & 789 & 546 & 923 \\
\hline & ref & 13 & 12 & 14 & 793 & 781 & 805 \\
\hline & bw & 13 & 10 & 15 & 653 & 372 & 923 \\
\hline & in total & 13 & 10 & 15 & 688 & 372 & 923 \\
\hline \multirow{5}{*}{ TRIESTE } & 3 & 17 & 14 & 20 & 42 & 22 & 60 \\
\hline & 5 & 14 & 11 & 16 & 122 & 17 & 232 \\
\hline & ref & 13 & 11 & 15 & 36 & 17 & 55 \\
\hline & bw & 16 & 13 & 20 & 94 & 22 & 232 \\
\hline & in total & 15 & 11 & 20 & 82 & 17 & 232 \\
\hline \multirow{5}{*}{ VENEZIA } & 5 & 4 & 3 & 5 & 6 & 4 & 9 \\
\hline & 10 & 5 & 4 & 5 & 6 & 4 & 8 \\
\hline & ref & 5 & 5 & 5 & 9 & 8 & 9 \\
\hline & bw & 4 & 3 & 5 & 5 & 4 & 6 \\
\hline & in total & 4 & 3 & 5 & 6 & 4 & 9 \\
\hline \multirow{5}{*}{ ANCONA } & 7 & 4 & 3 & 5 & 46 & 15 & 95 \\
\hline & 10 & 5 & 4 & 5 & 52 & 21 & 104 \\
\hline & ref & 4 & 4 & 4 & 100 & 95 & 104 \\
\hline & bw & 5 & 3 & 5 & 24 & 15 & 30 \\
\hline & in total & 4 & 3 & 5 & 49 & 15 & 104 \\
\hline \multirow{5}{*}{ BARI } & 5 & 13 & 13 & 14 & 113 & 60 & 164 \\
\hline & 11 & 14 & 13 & 15 & 132 & 109 & 175 \\
\hline & ref & n.a. & n.a. & n.a. & n.a. & n.a. & n.a. \\
\hline & bw & n.a. & n.a. & n.a. & n.a. & n.a. & n.a. \\
\hline & in total & 14 & 13 & 15 & 122 & 60 & 175 \\
\hline
\end{tabular}

stations directly impacted by BW discharge (further in text: impacted stations), and in six ports also at control stations (referent or anchorage sites). 18 taxa were observed only at impacted, and remaining 26 taxa at both impacted and control stations. Not a single taxa was observed exclusively at control station.

From a total of 62 sediment samples collected during the Adriatic PBS in nine ports, 40 distinct dinoflagellate taxa were identified (Table 4). Besides, a group of round brown cysts and small round cysts were distinctive from other unidentified cysts. In total, 29 taxa were identified to the species level, while other cysts were determined as a "group" (i.e., Alexandrium minutum/affine/tamutum, and A. tamarense/ catenella, and Diplopsalis), several to the genus and one to the family level (Thoracosphaeraceae). As many as twenty species belonged to the following three genera, Protoperidinium, Scrippsiella and Alexandrium, (nine, eight and three, respectively). The two most abundant taxa, Lingulodinium polyedra and A. minutum/affine/tamutum group, were also the only two taxa that were observed in all nine investigated ports.

Presence of vegetative form of each observed cyst taxa in each of investigated ports during PBS, which is presented in separate paper of this special issue (Mozetič et al., this issue), was checked and findings included in Table 4 (grey fields). Out of 40 distinct dinoflagellate taxa and three unidentified groups, 21 was observed in cyst and vegetative form, 14 at both impacted and control stations and 7 only at impacted 
Table 3

Average, minimal and maximal number of observed taxa (including unidentified groups) and cysts abundance $\left(\mathrm{g}^{-1} \mathrm{DW}\right.$ ) per port: (a) per month of sampling (two to three different months), (b) per type of sampling station (ballast water impacted - bw and referent stations - ref), and (c) all data included (- in total; all sampling station types and sampling months). Not available due to missing data (n.a.).

\begin{tabular}{|c|c|c|c|c|c|c|c|}
\hline \multirow[t]{2}{*}{ Port } & \multirow[t]{2}{*}{ Month } & \multicolumn{3}{|c|}{ Observed taxa } & \multicolumn{3}{|c|}{ Cysts abundance $\left(\mathrm{g}^{-1} \mathrm{DW}\right)$} \\
\hline & & Ave & Min & Max & Ave & Min & Max \\
\hline \multirow[t]{6}{*}{ Split } & 4 & 12 & 9 & 15 & 3399 & 252 & 11,554 \\
\hline & 6 & 7 & 7 & 7 & 680 & 680 & 680 \\
\hline & 10 & 8 & 8 & 8 & 1060 & 1060 & 1060 \\
\hline & ref & n.a. & n.a. & n.a. & n.a. & n.a. & n.a. \\
\hline & bw & n.a. & n.a. & n.a. & n.a. & n.a. & n.a. \\
\hline & in total & 10 & 7 & 15 & 2556 & 252 & 11554 \\
\hline \multirow[t]{5}{*}{ Šibenik } & 6 & 7 & 3 & 10 & 2745 & 610 & 4880 \\
\hline & 11 & 6 & 5 & 7 & 350 & 300 & 400 \\
\hline & ref & n.a. & n.a. & n.a. & n.a. & n.a. & n.a. \\
\hline & bw & n.a. & n.a. & n.a. & n.a. & n.a. & n.a. \\
\hline & In total & 6 & 3 & 10 & 1547 & 300 & 4880 \\
\hline \multirow[t]{5}{*}{ Rijeka } & 4 & 7 & 5 & 10 & 130 & 64 & 204 \\
\hline & 12 & 7 & 4 & 9 & 109 & 49 & 149 \\
\hline & ref & 7 & 7 & 7 & 89 & 64 & 113 \\
\hline & bw & 7 & 4 & 10 & 130 & 49 & 204 \\
\hline & In total & 7 & 4 & 10 & 120 & 49 & 204 \\
\hline \multirow[t]{5}{*}{ Pula } & 5 & 8 & 6 & 10 & 328 & 80 & 516 \\
\hline & 12 & 7 & 5 & 10 & 330 & 44 & 763 \\
\hline & ref & 8 & 7 & 8 & 336 & 224 & 449 \\
\hline & bw & 7 & 5 & 10 & 326 & 44 & 763 \\
\hline & In total & 7 & 5 & 10 & 329 & 44 & 763 \\
\hline \multirow[t]{5}{*}{ Koper } & 6 & 13 & 10 & 15 & 588 & 372 & 805 \\
\hline & 11 & 12 & 10 & 14 & 789 & 546 & 923 \\
\hline & ref & 13 & 12 & 14 & 793 & 781 & 805 \\
\hline & bw & 13 & 10 & 15 & 653 & 372 & 923 \\
\hline & In total & 13 & 10 & 15 & 688 & 372 & 923 \\
\hline \multirow[t]{5}{*}{ Trieste } & 3 & 17 & 14 & 20 & 42 & 22 & 60 \\
\hline & 5 & 14 & 11 & 16 & 122 & 17 & 232 \\
\hline & ref & 13 & 11 & 15 & 36 & 17 & 55 \\
\hline & bw & 16 & 13 & 20 & 94 & 22 & 232 \\
\hline & In total & 15 & 11 & 20 & 82 & 17 & 232 \\
\hline \multirow[t]{5}{*}{ Venezia } & 5 & 4 & 3 & 5 & 6 & 4 & 9 \\
\hline & 10 & 5 & 4 & 5 & 6 & 4 & 8 \\
\hline & ref & 5 & 5 & 5 & 9 & 8 & 9 \\
\hline & bw & 4 & 3 & 5 & 5 & 4 & 6 \\
\hline & In total & 4 & 3 & 5 & 6 & 4 & 9 \\
\hline \multirow[t]{5}{*}{ Ancona } & 7 & 4 & 3 & 5 & 46 & 15 & 95 \\
\hline & 10 & 5 & 4 & 5 & 52 & 21 & 104 \\
\hline & ref & 4 & 4 & 4 & 100 & 95 & 104 \\
\hline & bw & 5 & 3 & 5 & 24 & 15 & 30 \\
\hline & In total & 4 & 3 & 5 & 49 & 15 & 104 \\
\hline \multirow[t]{5}{*}{ Bari } & 5 & 13 & 13 & 14 & 113 & 60 & 164 \\
\hline & 11 & 14 & 13 & 15 & 132 & 109 & 175 \\
\hline & ref & n.a. & n.a. & n.a. & n.a. & n.a. & n.a. \\
\hline & bw & n.a. & n.a. & n.a. & n.a. & n.a. & n.a. \\
\hline & In total & 14 & 13 & 15 & 122 & 60 & 175 \\
\hline
\end{tabular}

ones, whereas 22 were present only in cyst form, one half being observed only at impacted and the other at both type of stations.

A review of earlier findings of each taxa identified during this PBS endeavour, in the Adriatic and further afield, although primarily in the Mediterranean, is presented in Table 5. Based on existing/available literature, we established that out of 40 observed taxa in this PBS (Table 6):

1) six were assumed as new introductions for the Adriatic as no previous reports of observation were available for any area in the Adriatic: Alexandrium affine, found in ports of Rijeka and Pula, Alexandrium insuetum and Alexandrium margaleffii, found in port of Trieste, Polykrikos hartmanii, found in ports of Bari, Rijeka and Pula, Preperidinium meunieri, found in ports of Bari, Rijeka and Split, and
Scrippsiella cf. rotunda, found in ports of Trieste and Koper; and

2) eleven were observed in the port/s endorsed in area/s where until now no observation were reported, although were reported in at least one other area of the Adriatic: Alexandrium tamarense/catenella group, Biecheleria spp., Cochlodinium spp., Gonyaulax scrippsae, Gymnodinium impudicum, Protoceratium reticulatum, Protoperidinium compressum, Protoperidinium conicoides, Protoperidinium leonis, Protoperidinium pentagonum, and Scrippsiella precaria.

Description of each port regarding seasonal aspect and station types, based on integral dataset (not shown), follows and is described in Fig. 2.

Split-Samplings in this port were performed in April 2011, October 2011 and June 2014. In this port no control location was investigated. Overall, cyst abundance was highest in this port. PBS maximum was reported in April (station STbwK1, 11,554 $\mathrm{g}^{-1} \mathrm{DW}$ ) and third highest PBS abundance in October (STbwJ1, $1060 \mathrm{~g}^{-1} \mathrm{DW}$ ). Cyst diversity in April (19 taxa) was considerably higher than in October (8) and June (7). However, four stations were investigated in April (in each 9, 11, 12 and 15 taxa were determined, 19 in total), and only one per other two occasions. As two stations (STbwK1 and STbwJ1) sampled in April were resampled in October and June, diversity can be more closely compared: decrease from 15 to 7 taxa, and 9 to 8, respectively. The most abundant cyst was L. polyedra (contribution of 85-86\%) in April and October, which evidently dominated the cyst assemblage and was present in bloom abundances. In June, contribution of $L$. polyedra decreased to $(26 \%)$, and co-dominated with undetermined cysts of genus Gymnodinium (44\%) and cysts identified as group A. minutum/affine/ tamutum (12\%). During this PBS, Protoperidinium pentagonum was found only in port of Split, at three out of four investigated influenced locations in April.

Šibenik - Two PBS samplings were performed in November 2013 and June 2014. In this port no control location was investigated. At influenced locations, cyst abundance and diversity were considerably higher in June than in previous November. The second highest abundance during PBS performed in all nine ports was observed in this port, in June 2014 at sampling station SIbw1, due to especially high abundance of cysts identified as group A. minutum/affine/tamutum $\left(4800 \mathrm{~g}^{-1}\right.$ DW). This group was dominant in November (53\%), with undetermined cysts of genus Polykrikos (14\%), S. acuminata (13\%) and A. tamarense/ catenella $(11 \%)$, and again in extreme amount in June, contributing with high $91 \%$ in total abundance. No species were observed solely in this port.

Rijeka - During the two PBS samplings, in December 2014 and May 2015, cyst diversity remained similar, even at sampling location level, around averages of PBS, while abundances slightly decreased at influenced locations and increased at the control one. In December, the most abundant cysts at control were L. polyedra (50\%), undetermined cysts of genus Biecheleria (32\%) and Gymnodinium (12\%). At impacted stations, the same taxa were dominant $(17$, nine and $41 \%$, respectively), including also undetermined cysts of genus Protoperidinium (12\%). Dominant taxa slightly changed during May, L. polyedra (57\%), cysts identified as group A. minutum/affine/tamutum (17\%) and unidentified cysts $(13 \%)$ at control location, and undetermined cysts of genus Protoperidinium (28\%) and Biecheleria (16\%), L. polyedra (12\%) and unidentified cysts $(20 \%)$ at impacted locations. No species were observed solely in this port.

Pula - During the two PBS samplings, in December 2014 and May 2015, cyst diversity and abundances remained similar, even at sampling type level. In December, the most abundant cysts at control location were undetermined cysts of genus Protoperidinium (33\%), L. polyedra (32\%), Polykrikos hartmanii $(20 \%)$ and generally undetermined cysts (15\%), while at influenced locations, L. polyedra with $76 \%$ was predominant. In May, diversity was more uniform across the port, with $L$. polyedra (53\%) and generally undetermined cysts (16\%). No species were observed solely in this port. 


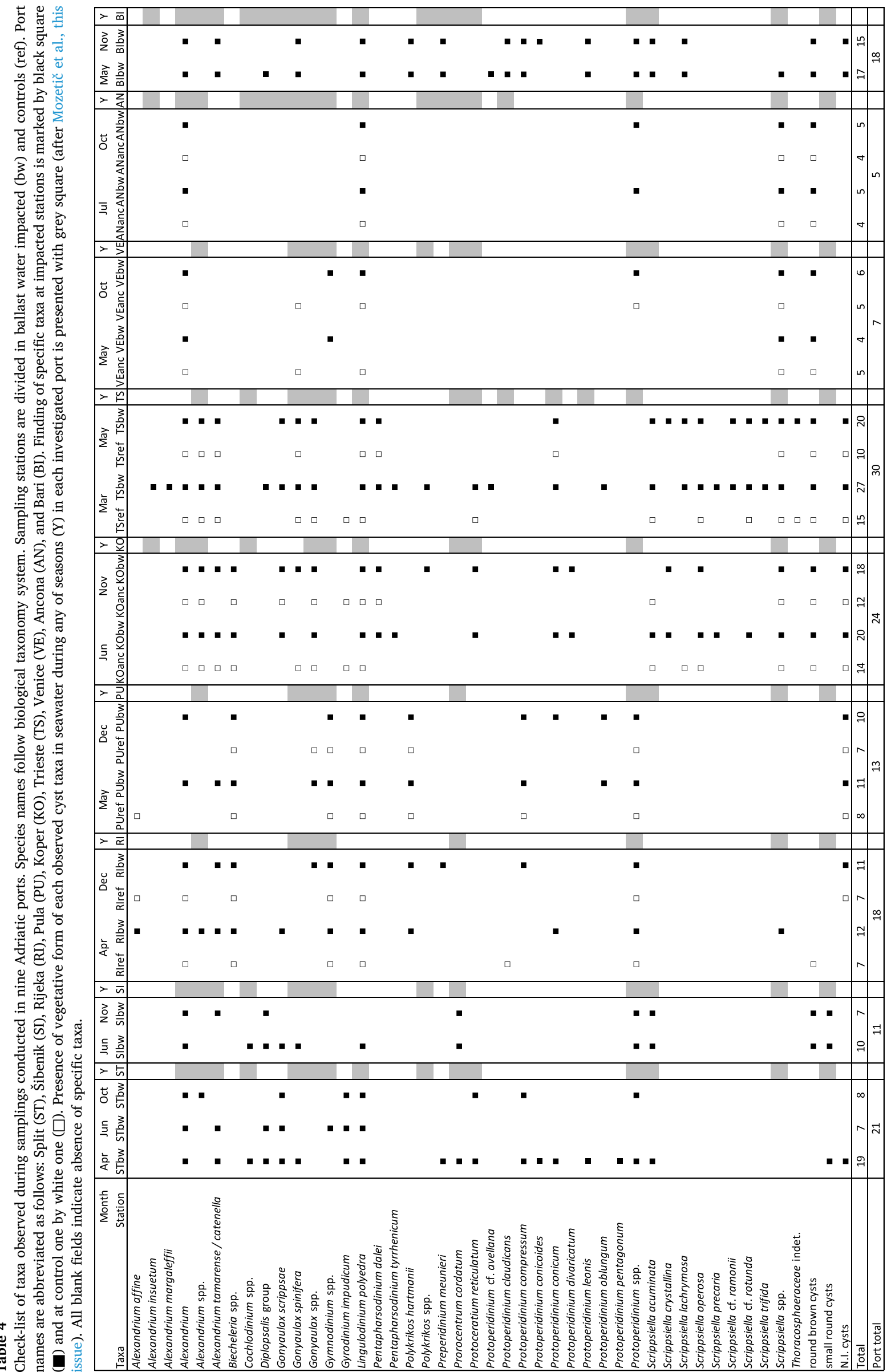


Table 5

Check-list of the taxa observed during PBS in the nine Adriatic Sea. Taxa are named after biological taxonomy system (after global algal database of taxonomic, nomenclatural and distributional information, AlgaeBase; www.algaebase.com) including biological and paleontological synonyms. Underlined taxa are (potentially) toxic; basic information about toxicity is included. Taxa distribution is reported for: Europe/European Seas (source: AlgaeBase), Mediterranean Sea (All) including sub-basins: Alboran (Alb), Algerian (Alg), Balear-Provencal (Bal), Ligurian (Lig), Tyrrhenian (Tyr), Ionian (Ion), Aegean (Aeg), Levantine (Lev), Marmara (Mar) and Adriatic (Adr), after Gómez (2003), and the Adriatic Sea, divided in six areas: south-eastern (SE), mid-eastern (mE) - endorsing area of ports of Split and Šibenik; north-eastern (NE) - endorsing area of ports of Rijeka, Pula, Koper and Trieste; north-western (NW) - endorsing area of port Venice; mid-western (mW) - endorsing area of port Ancona; and south-western (SW) - endorsing area of port Bari.

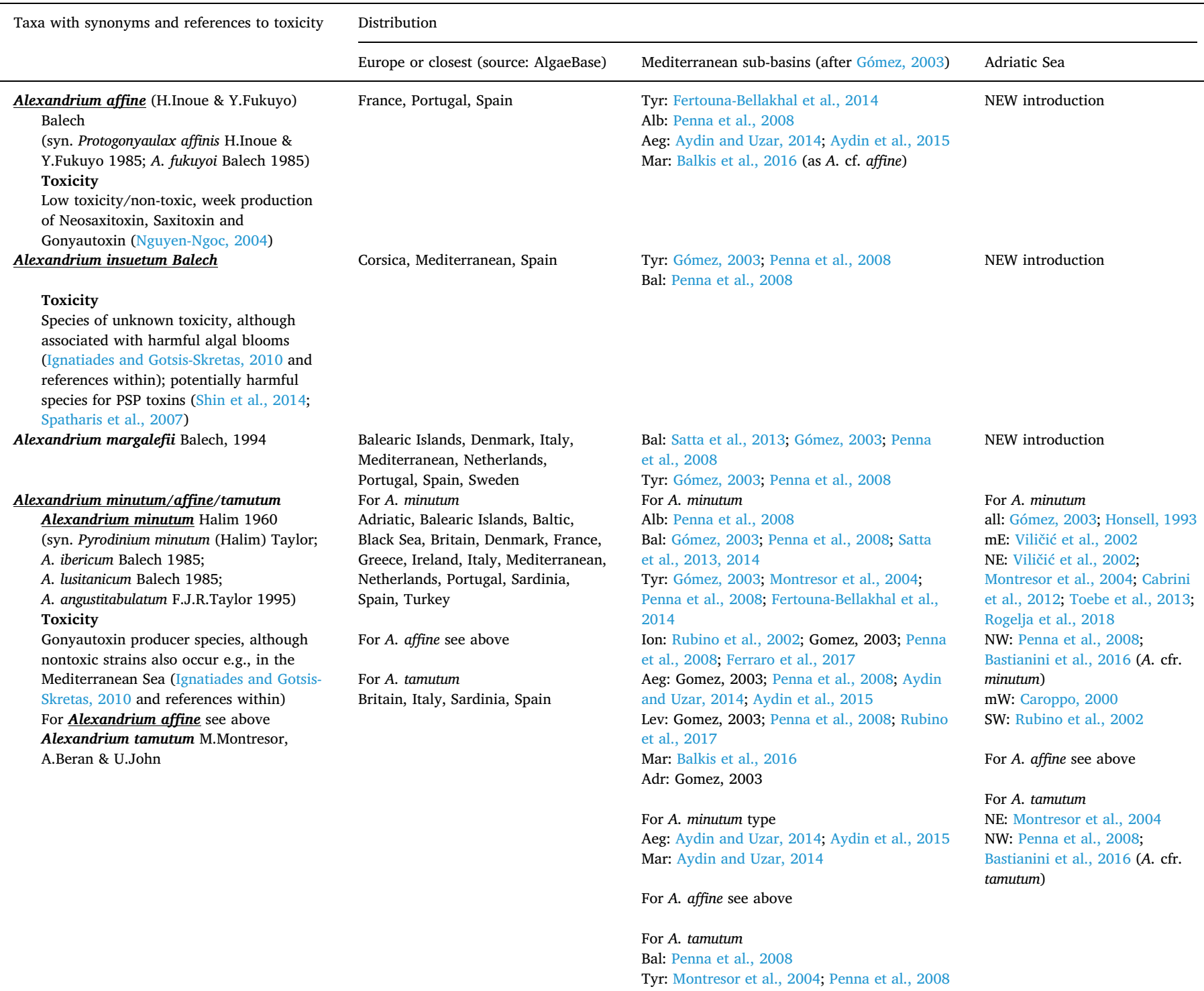


Table 5 (continued)

\author{
Taxa with synonyms and references to toxicity \\ Alexandrium tamarense/catenella \\ Alexandrium tamarense (Lebour) Balech \\ 1995 \\ (syn. Gonyaulax tamarensis Lebour 1925; \\ Gessnerium tamarensis (Lebour) Loeblich III \\ \& L.Loeblich 1979; \\ Protogonyaulax tamarensis (Lebour) \\ F.J.R.Taylor 1979; \\ Gonyaulax tamarensis var. excavata \\ T.Braarud 1945; \\ Gonyaulax excavata (Braarud) Balech \\ 1971; \\ Protogonyaulax excavata (Braarud) \\ F.J.R.Taylor 1979; \\ A. excavatum (Braarud) Balech \& Tangen \\ 1985; \\ A. tamarense f. excavatum (T.Braarud) \\ G.V.Konovalova 1993) \\ Toxicity \\ Gonyautoxin producer species (Ignatiades \\ and Gotsis-Skretas, 2010 and references \\ within) \\ Alexandrium catenella (Whedon \& \\ Kofoid) Balech \\ (syn. Gonyaulax catenella Whedon \& Kofoid \\ 1936; \\ Protogonyaulax catenella (Whedon \& \\ Kofoid) Taylor 1979; \\ Gessnerium catenella (Whedon \& Kofoid) \\ F.J.R.Taylor 1979; \\ Gonyaulax washingtonensis Hsu 1967; \\ A. fundyense Balech 1985) \\ Toxicity \\ Harmful algal bloom species, paralytic \\ shellfish poisoning (PSP) toxins-producing \\ species (Anderson et al., 2012 and \\ references within), c1-c4 toxins, Saxitoxins \\ and Gonyautoxins producer (Ignatiades \\ and Gotsis-Skretas, 2010 and references \\ within) \\ Alexandrium spp. Halim, 1960
}

\begin{abstract}
Biecheleria spp. Moestrup, Lindberg \&
Daugbjerg, 2009
\end{abstract}

Cochlodinium spp. F.Schütt, 1896

Diplopsalis group R.S.Bergh, 1881
Distribution

Europe or closest (source: AlgaeBase)

Balearic Islands, Baltic Sea, Black Sea,

Britain, Faroe Islands, France, Greece,

Ireland, Italy, Mediterranean,

Netherlands, Norway, North sea,

Portugal, Sardinia, Spain, UK (as A.

tamarense)

Netherlands

(as Gonyaulax tamarensis)

Britain

(as Gonyaulax excavata, G. catenella and G. tamarensis)

France, Greece, Mediterranean, Sardinia, Spain (as A. catenella)
All: Gómez, 2003Bal: Penna et al., 2008; Satta et al., 2013, 2014

Tyr: Montresor et al., 1998; Penna et al., 2008;

Fertouna-Bellakhal et al., 2014

Ion: Penna et al., 2008; Ferraro et al., 2017

Aeg: Penna et al., 2008

Lev: Penna et al., 2008; Rubino et al., 2017

Mar: Balkis et al., 2016

Bal: Satta et al., 2013, 2014 (as B. cincta)

Alb, Tyr, Lig, Adr, Lev: Gómez, 2003

Bal: Gómez, 2003; Satta et al., 2013 (as C. polykrikoides)

Ion: Gómez, 2003; Ferraro et al., 2017 (as C. polykrikoides)

All: Gómez, 2003 (as D. lenticula); Zonneveld

et al., 2013 (as Brigantedinium sp.)

Tyr: Montresor et al., 1998; Fertouna-Bellakhal

et al., 2014 (as D. lenticula)

Ion: Rubino et al., 2002; Ferraro et al., 2017 (as D. lenticula)

Lev: Rubino et al., 2017 (as D. lenticula)

Mar: Balkis et al., 2016
Adriatic Sea

For A. tamarense

All: Zonneveld et al., 2013

NE: Rogelja et al., 2018

NW: Sangiorgi and Donders,

2004 (cf. A. tamarense type)

For $A$. cf. tamarense

NE: Nichetto et al., 1995

SW: Rubino et al., 2002

For A. catenella

All: Gomez, 2003

For A. fundyense

SE: Saracino and Rubino,

2006b; Rubino et al., 2009
SE: Saracino and Rubino, 2006b mE: Totti et al., 2000

NE: Cabrini et al., 2012; Rogelja et al., 2018

NW: Penna et al., 2008;

Bastianini et al., 2016

mW: Caroppo, 2000; Totti et al., 2000

SW: Caroppo et al., 1999;

Rubino et al., 2009

NW: Bastianini et al., 2016

SE: Saracino and Rubino, 2006b; Rubino et al., 2009

NE: Cabrini et al., 2012; Rogelja et al., 2018 (as C. polykrikoides)

SW: Rubino et al., 2009

All: Gómez, 2003 (as D. lenticula); Zonneveld et al., 2013

(as Brigantedinium sp.)

SE: Viličić et al., 2002;

Drakulović et al., 2012 (as $D$.

lenticula)

mE: Viličić et al., 2002

NE: Nichetto et al., 1995; Viličić et al., 2002; Cabrini et al., 2012; Rogelja et al., 2018 (as D.

lenticula)

mW: Caroppo, 2000

SW: Caroppo et al., 1999;

Rubino et al., 2002

(continued on next page) 
Table 5 (continued)

\begin{tabular}{|c|c|c|}
\hline \multirow[t]{2}{*}{ Taxa with synonyms and references to toxicity } & \multicolumn{2}{|l|}{ Distribution } \\
\hline & Europe or closest (source: AlgaeBase) & Mediterranean sub-basins (after Gómez, 2003) \\
\hline $\begin{array}{l}\text { Gonyaulax scrippsae Kofoid } \\
\text { (syn. Spiniferites bulloideus (Deflandre \& } \\
\text { Cookson) Sargeant) }\end{array}$ & $\begin{array}{l}\text { Black Sea, Britain, Mediterranean, } \\
\text { Netherlands, Portugal }\end{array}$ & $\begin{array}{l}\text { Bal, Lig: Gómez, } 2003 \\
\text { Tyr: Gómez, 2003; Montresor et al., 1998; } \\
\text { Fertouna-Bellakhal et al., } 2014 \\
\text { Ion: Rubino et al., 2002; Gómez, 2003; } \\
\text { Zonneveld et al., } 2013 \text { (as Spiniferites sp.) } \\
\text { Aeg: Aydin and Uzar, 2014; Aydin et al., } 2015 \\
\text { Lev: Gomez, 2003; Aydin and Uzar, 2014 } \\
\text { Mar: Aydin and Uzar, } 2014\end{array}$ \\
\hline
\end{tabular}

\section{Gonyaulax spinifera}

(Claparède \& Lachmann) Diesing (syn. Peridinium spiniferum Claparède \& Lachmann 1859;

Peridinium levanderi Lemmermann; Spiniferites ramosus (Ehrenberg) Mantell; G. levanderi (Lemmermann) Paulsen 1907; Tectatodinium pellitum Wall 1967)

Toxicity

Yessotoxin and Homoyessotoxin producer (Rhodes et al., 2006; Riccardi et al., 2009)

\section{Gonyaulax spp. Diesing, 1866}

(syn. Spiniferites spp.)
Adriatic Sea, Baltic Sea, Black Sea, Britain, Georgia, Greece, Helgoland, Ireland, Mediterranean, Netherlands, Portugal, Romania, Sweden
All: Gómez, 2003; Zonneveld et al., 2013 (as Spiniferites $\mathrm{sp.)}$

Tyr: Fertouna-Bellakhal et al., 2014

Ion: Rubino et al., 2002

Aeg: Aydin et al., 2015 (as G. spinifera complex)

Mar: Aydin and Uzar, 2014 (as S. ramosus); Balkis et al., 2016

All: Gómez, 2003; Zonneveld et al., 2013 (as Spiniferites sp.)

Bal: Satta et al., 2013, 2014

Tyr: Montresor et al., 1998; Fertouna-Bellakhal et al., 2014

Ion: Ferraro et al., 2017

Aeg: Aydin and Uzar, 2014; Aydin et al., 2015 Lev: Aydin and Uzar, 2014; Rubino et al., 2017 Mar: Aydin and Uzar, 2014; Balkis et al., 2016

Alb, Tyr, Adr: Gómez, 2003

Bal: Gómez, 2003; Satta et al., 2014

Ion: Gómez, 2003; Ferraro et al., 2017

Portugal

(as Gyrodinium impudicum)

Lev: Gómez, 2003; Rubino et al., 2017
All: Gómez, 2003

Bal: Satta et al., 2013, 2014

Tyr: Montresor et al., 1998

Ion: Rubino et al., 2002; Ferraro et al., 2017

Aeg: Aydin et al., 2015

Lev: Rubino et al., 2017

Mar: Balkis et al., 2016
Adriatic Sea

All: Zonneveld et al., 2013 (as

Spiniferites sp.)

NE: Nichetto et al., 1995;

Rubino et al., 2000 (as $S$.

bulloideus); Cabrini et al., 2012

NW: Sangiorgi and Donders,

2004 (as S. bulloideus); Rubino

et al., 2000 (as S. bulloideus)

mW: Rubino et al., 2000 (as $S$.

bulloideus)

SW: Rubino et al., 2002

All: Gómez, 2003; Zonneveld

et al., 2013 (as Spiniferites sp.)

SE: Saracino and Rubino,

2006b; Rubino et al., 2009;

Drakulović et al., 2012

mE: Viličić et al., 2002

NE: Viličić et al., 2002; Cabrini et al., 2012

NW: Tolomio and Bullo, 2001; Sangiorgi and Donders, 2004; Bastianini et al., 2016 (also cyst) mW: Caroppo, 2000

SW: Caroppo et al., 1999; Rubino et al., 2002, 2009 All: Zonneveld et al., 2013 (as Spiniferites sp.)

SE: Drakulović et al., 2012

mE: Totti et al., 2000

NE: Nichetto et al., 1995; Viličić et al., 2009; Cabrini et al., 2012; Rogelja et al., 2018

NW: Sangiorgi and Donders, 2004; Bastianini et al., 2016 mW: Caroppo, 2000; Totti et al., 2000

SW: Caroppo et al., 1999

all: Gómez, 2003

NE: Rogelja et al., 2018
All: Gómez, 2003

SE: Viličić et al., 2002; Saracino and Rubino, 2006b; Rubino et al., 2009; Cabrini et al., 2012; Drakulović et al., 2012 mE: Totti et al., 2000; Viličić et al., 2002; Cabrini et al., 2012 NE: Nichetto et al., 1995; Rubino et al., 2000; Viličić et al., 2002; Bernardi Aubry et al., 2004; Viličić et al., 2009; Cabrini et al., 2012; Rogelja et al., 2018

NW: Rubino et al., 2000; Bernardi Aubry et al., 2004; Sangiorgi and Donders, 2004; Bastianini et al., 2016 mW: Caroppo, 2000; Rubino et al., 2000; Totti et al., 2000 SW: Caroppo et al., 1999; Rubino et al., 2002, 2009

(continued on next page) 
Table 5 (continued)

\begin{tabular}{ll}
\hline Taxa with synonyms and references to toxicity & Distribution \\
\cline { 2 - 2 } & Europe or closest (source: AlgaeBase) \\
\hline $\begin{array}{ll}\text { Lingulodinium polyedra } \text { (F.Stein) J.D.Dodge } \\
\text { (syn. Gonyaulax polyedra F.Stein 1883; }\end{array}$ & $\begin{array}{l}\text { Adriatic Sea, Baltic Sea, Black Sea, } \\
\text { Croatia, Mediterranean, Netherlands, }\end{array}$ \\
$\begin{array}{l}\text { W. machaerophorum (Deflandre \& Cookson) } \\
\text { Woxicity }\end{array}$ & $\begin{array}{l}\text { Portugal, Romania, Spain, Sweden, } \\
\text { Turkey }\end{array}$ \\
$\begin{array}{l}\text { Saxitoxin (Bates et al., 1978; Bruno et al., } \\
\text { 1990), Yessotoxin and probably }\end{array}$ & $\begin{array}{l}\text { Britain, Ireland, Netherlands, } \\
\text { Homoyessotoxin producer (Yasumoto and } \\
\text { Satake, 1998; Paz et al., 2004) }\end{array}$ \\
$\begin{array}{l}\text { (as G. polyedra) } \\
\end{array}$
\end{tabular}

Mediterranean sub-basins (after Gómez, 2003)

All: Gómez, 2003; Zonneveld et al., 2013

Bal: Satta et al., 2013

Tyr: Montresor et al., 1998; Fertouna-Bellakhal et al., 2014

Ion: Rubino et al., 2002; Ferraro et al., 2017 Aeg: Aydin and Uzar, 2014; Aydin et al., 2015 Lev: Aydin and Uzar, 2014; Rubino et al., 2017 Mar: Aydin and Uzar, 2014; Balkis et al., 2016
Pentapharsodinium dalei Indelicato \& Loeblich

Pentapharsodinium tyrrhenicum (Balech) Montresor, Zingone \& Marino (syn. Peridinium tyrrhenicum Balech; Calcicarpinum bivalvum Versteegh)

Polykrikos hartmannii W.Zimmermann (syn. Pheopolykrikos hartmannii (W.Zimmermann) Matsuoka \& Fukuyo 1986)

Polykrikos spp. Bütschli, 1873 Possibly including:

Polykrikos schwartzii Bütschli 1873 Polykrikos kofoidii Chatton 1914 (syn. Polykrikos schwarzii Kofoid)
Baltic Sea, Black Sea, Britain, Netherlands, Sweden

Black Sea, Italy, Mediterranean

Black Sea, Mediterranean, Netherlands, Portugal (as Pheopolykrikos hartmannii)

Baltic Sea, Black Sea, Britain, Greece, Helgoland, Mediterranean, Netherlands, Portugal, Turkey (as P. schwarzii)

Adriatic Sea, Black Sea, Britain, Germany, Greece, Helgoland, Mediterranean, Netherlands, Portugal (as P. kofoidii)

Romania

(as P. schwarzii)
All: Zonneveld et al., 2013

Bal: Satta et al., 2013, 2014

Ion: Rubino et al., 2002; Ferraro et al., 2017

Lev: Rubino et al., 2017

All: Zonneveld et al., 2013

Bal: Satta et al., 2013, 2014

Ion: Rubino et al., 2002; Ferraro et al., 2017 Tyrrhenian: Montresor et al., 1993, 1994,

1998; Gomez, 2003; Gottschling et al., 2005 Lev: Rubino et al., 2017

Tyr: Gómez, 2003; Fertouna-Bellakhal et al. 2014

Lev: Rubino et al., 2017

\section{For P. schwarzii:}

Alb, Bal, Lig: Gómez, 2003

Tyr: Montresor et al., 1998; Gómez, 2003;

Zonneveld et al., 2013

Ion: Rubino et al., 2002; Zonneveld et al., 2013

Mar: Zonneveld et al., 2013; Balkis et al., 2016

Aeg: Zonneveld et al., 2013; Aydin and Uzar,

2014; Aydin et al., 2015

Adr: Zonneveld et al., 2013

For P. kofoidii

Bal, Ion, Lig: Gomez, 2003

Alg: Zonneveld et al., 2013

Alb, Adr: Gomez, 2003; Zonneveld et al., 2013

Tyr: Montresor et al., 1998; Gomez, 2003;

Zonneveld et al., 2013; Fertouna-Bellakha

et al., 2014

Aeg: Gomez, 2003; Zonneveld et al., 2013;

Aydin and Uzar, 2014; Aydin et al., 2015

Mar: Aydin and Uzar, 2014; Balkis et al., 2016

For $P$. schwartzii and $P$. kofoidii:

Ion: Ferraro et al., 2017

For P. schwartzii/kofoidii:

Lev: Rubino et al., 2017

For Polykrikos sp.

Bal: Satta et al., 2013
Adriatic Sea

All: Gómez, 2003; Zonneveld

et al., 2013

SE: Rubino et al., 2002; Viličić et al., 2002; Saracino and

Rubino, 2006b; Rubino et al., 2009

mE: Totti et al., 2000; Viličić et al., 2002

NE: Nichetto et al., 1995 (as G. polyedra); Rubino et al., 2000; Viličić et al., 2002, 2009; Cabrini et al., 2012, Rogelja et al., 2018 NW: Rubino et al., 2000; Tolomio and Bullo, 2001; Sangiorgi and Donders, 2004; Bastianini et al., 2016 mW: Caroppo, 2000; Rubino et al., 2000; Totti et al., 2000 SW: Caroppo et al., 1999; Rubino et al., 2002 All: Zonneveld et al., 2013

NE: Rogelja et al., 2018 NW: Sangiorgi and Donders, 2004

SW: Rubino et al., 2002 All: Zonneveld et al., 2013 SE: Saracino and Rubino, 2006b; Rubino et al., 2009 NE: Rogelja et al., 2018 SW: Rubino et al., 2002

NEW introduction

All: Gómez, 2003; Zonneveld et al., 2013 (P. kofoidii) All: Zonneveld et al., 2013 (P. schwarzii)

SE: Saracino and Rubino, 2006b; Rubino et al., 2009 (P. schwarzii)

mE: Viličić et al., 2002 (P. kofoidii)

NE: Nichetto et al., 1995; Rubino et al., 2000; Rogelja et al., 2018 (P. schwarzii) NW: Rubino et al., 2000; Sangiorgi and Donders, 2004 (P. schwarzii)

mW: Rubino et al., 2000; Zonneveld et al., 2013 (P. schwarzii)

SW: Caroppo et al., 1999 ( $P$. kofoidii); Rubino et al., 2002, 2009; Zonneveld et al., 2013 (P. schwarzii) 
Table 5 (continued)

Taxa with synonyms and references to toxicity

Preperidinium meunieri (Pavillard) Elbrächte (syn. Peridinium meunieri Pavillard 1912; Diplopsalis lenticula f. minor Paulsen 1907; Peridinium lenticulum Mangin 1911; Peridinium paulsenii Mangin 1911; Peridinium lenticulatum L.A.Mangin 1911; Diplopeltopsis minor Pavillard 1913; Preperidinium paulseni (Mangin) Mangin 1913;

Diplopsalis minor (Paulsen) Lindemann 1927;

Glenodinium lenticula f. minor Schiller 1937; Zygabikodinium lenticulatum Loeblich Jr. \& Loeblich III 1970)

Protoceratium reticulatum (Claparède \& Lachmann) Bütschli

(syn. Peridiniopsis reticulatum (Claparède \& Lachmann) Starmach;

Peridinium reticulatum Claparède \&

Lachmann 1859;

Gonyaulax grindleyi Reinecke 1967;

Operculodinium centrocarpum

(Deflandre and Cookson) Wall)

\section{Toxicity}

Yessotoxin toxin producer (MacKenzie et al., 1998; Paz et al., 2004; Samdal et al., 2004; Miles et al., 2005a, 2005b; Paz et al., 2006; Loader et al., 2007; Ignatiades and Gotsis-Skretas, 2010 and references within)

Protoperidinium cf. avellana (Meunier) Balech (syn. Brigantedinium cariacoense (Wall) Lentin and Williams)

Protoperidinium claudicans (Paulsen) Balech (syn. Peridinium claudicans Paulsen 1907; Votadinium spinosum Reid)

Protoperidinium compressum (Abé) Balech (syn. Peridinium compressum (Abé) Nie; Stelladinium reidii Bradford)

Protoperidinium conicoides (Paulsen) Balech (syn. Peridinium conicoides Paulsen; Brigantedinium simplex (Wall) P.C.Reid)

Protoperidinium conicum (Gran) Balech (syn. Peridinium divergens var. conica Gran 1900;

Peridinium conicum (Gran) Ostenfeld \& Schmidt 1902;

Selenopemphix quanta (M.R.Bradford) Matsuoka 1985)

Distribution

Europe or closest (source: AlgaeBase)

Mediterranean sub-basins (after Gómez, 2003)

Adriatic Sea

Baltic Sea, Black Sea, Helgoland,

Lig, Lev: Gómez, 2003

NEW introduction

Mediterranean, Netherlands, Portugal

Bal: Gómez, 2003; Satta et al., 2013 (as P. cf. meunieri)

Spain

(as D. lenticula f. minor)

Netherlands

(as Diplopeltopsis minor)

Britain, Netherlands, Norway,

Sweden

(as Z. lenticulatum)

Adriatic Sea, Baltic Sea, Black Sea, Helgoland, Mediterranean, Netherlands, Romania, Sweden (as $P$. reticulatum)

Romania

(as Peridiniopsis reticulatum)

Adriatic Sea, Black Sea, Britain, Portugal, Turkey

(as G. grindleyi)

Baltic Sea, Britain, Portugal (as P. avellana)

Baltic Sea, Black Sea, Britain, Helgoland, Mediterranean, Netherlands, Portugal, Turkey

South-west Asia: Turkey

Baltic Sea, Black Sea, Britain, Mediterranean, Netherlands, Portugal, Sweden

Adriatic Sea, Baltic Sea, Black Sea, Britain, France, Greece, Helgoland, Mediterranean, Netherlands, Portugal, Sweden, Turkey
All: Gómez, 2003; Zonneveld et al., 2013

Bal: Satta et al., 2013

Tyr: Montresor et al., 1998; Fertouna-Bellakhal et al., 2014

Ion: Rubino et al., 2002

Aeg: Aydin and Uzar, 2014; Aydin et al., 2015 Lev: Aydin and Uzar, 2014

Mar: Aydin and Uzar, 2014; Balkis et al., 2016 all: Gómez, 2003; Zonneveld et al., 2013

NE: Nichetto et al., 1995 (as G. grindleyi); Viličić et al., 2002 NW: Sangiorgi and Donders, 2004; Bastianini et al., 2016 (also cyst)

SW: Rubino et al., 2002, 2009
All: Zonneveld et al., 2013

Tyr: Montresor et al., 1998 (as P. avellanum) Aeg: Aydin et al., 2015

Bal: Gómez, 2003; Zonneveld et al., 2013 (as V. spinosum); Satta et al., 2013

Alb, Alg, Lig, Lev: Gómez, 2003

Tyr: Montresor et al., 1998; Gómez, 2003;

Fertouna-Bellakhal et al., 2014

Aeg: Gomez, 2003; Aydin and Uzar, 2014;

Aydin et al., 2015

Mar: Aydin and Uzar, 2014

Bal: Satta et al., 2013, 2014

Tyr: Montresor et al., 1998; Fertouna-Bellakhal et al., 2014

Ion: Rubino et al., 2002; Ferraro et al., 2017

Aeg: Evagelopoulos and Nikolaidis, 1996

Lev: Rubino et al., 2017

Mar: Aydin and Uzar, 2014; Balkis et al., 2016

All: Zonneveld et al., 2013

Tyr: Fertouna-Bellakhal et al., 2014

Aeg: Aydin and Uzar, 2014; Aydin et al., 2015

Lev: Gómez, 2003

Mar: Aydin and Uzar, 2014; Balkis et al., 2016

All: Gómez, 2003; Zonneveld et al., 2013 (as S.

quanta)

Bal: Satta et al., 2013

Tyr: Montresor et al., 1998; Fertouna-Bellakhal et al., 2014

Ion: Rubino et al., 2002; Ferraro et al., 2017

Aeg: Aydin and Uzar, 2014; Aydin et al., 2015

Lev: Rubino et al., 2017

Mar: Aydin and Uzar, 2014; Balkis et al., 2016
All: Zonneveld et al., 2013 (as Brigantedinium sp.) NW: Sangiorgi and Donders, 2004

NW: Sangiorgi and Donders, 2004; Bastianini et al., 2016

NE: Nichetto et al., 1995;

Rubino et al., 2000

NE: Nichetto et al., 1995; Rubino et al., 2000

NW: Rubino et al., 2000 mW: Rubino et al., 2000 SW: Rubino et al., 2002, 2009

All: Zonneveld et al., 2013 (as Brigantedinium sp.)

NW: Sangiorgi and Donders, 2004

all: Gómez, 2003; Zonneveld et al., 2013 (as S. quanta) SE: Viličić et al., 2002; Saracino and Rubino, 2006b mE: Viličić et al., 2002 NE: Rubino et al., 2000; Viličić et al., 2002; Cabrini et al., 2012; Rogelja et al., 2018

NW: Rubino et al., 2000; Sangiorgi and Donders, 2004; Bastianini et al., 2016 mW: Rubino et al., 2000 SW: Rubino et al., 2002

(continued on next page) 
Table 5 (continued)

Taxa with synonyms and references to toxicity

Protoperidinium divaricatum (Meunier) Balech

(syn. Peridinium divaricatum Meunier 1919;

Peridinium gainii P.-A.Dangeard 1920;

Protoperidinium gainii (P.-A.Dangeard) Balech 1974;

Xandarodinium xanthum Reid)

Protoperidinium leonis (Pavillard) Balech (syn. Peridinium leonis Pavillard 1916; Peridinium saltans Pavillard 1915; Quinquecuspis concreta (Reid) Head)

Protoperidinium oblongum (Aurivillius) Parke \& Dodge

(syn. Peridinium divergens var. oblongum Aurivillius 1898;

Peridinium oblongum (Aurivillius) Cleve

1900;

Peridinium oceanicum var. oblongum

(Aurivillius) Paulsen 1908;

Peridinium oceanicum f. oblongum

(Aurivillius) Broch 1910;

Votadinium calvum Reid 1977)

Protoperidinium pentagonum (Gran) Balech (syn. Peridinium pentagonum Gran 1902; Peridinium sinuosum Lemmermann 1905; Protoperidinium parapentagonum Wang 1936;

Trinovantedinium applanatum (Bradford) Bujak and Davies)

Protoperidinium spp. R.S.Bergh, 1881 (syn. Brigantedinium)

Distribution

Europe or closest (source: AlgaeBase)

Mediterranean sub-basins (after Gómez, 2003)

Adriatic Sea

Asia: East China Sea, Yellow Sea

Tyr: Montresor et al., 1998

Ion: Ferraro et al., 2017

Aeg: Zonneveld et al., 2013 (as X. xanthum)

Aydin and Uzar, 2014; Aydin et al., 2015

Adr: Zonneveld et al., 2013 (as X. xanthum)

Mar: Aydin and Uzar, 2014

Adriatic Sea, Baltic Sea, Black, Britain, France, Greece, Helgoland Mediterranean, Netherlands, Portugal, Sweden, Turkey 2013 et al., 2014
Baltic Sea, Black Sea, Britain, Helgoland, Ireland, Mediterranean, Netherlands, Portugal, Sweden

Baltic Sea, Black Sea, Britain, Helgoland, Mediterranean, Netherlands, Portugal, Romania, Turkey
Alb, Alg, Adr: Gómez, 2003; Zonneveld et al.,

Bal: Gómez, 2003; Zonneveld et al., 2013; Satta

Tyr: Montresor et al., 1998 (as P. leone) Gomez, 2003; Zonneveld et al., 2013; Fertouna-Bellakhal et al., 2014

Lig: Gomez, 2003

Ion: Zonneveld et al., 2013; Ferraro et al., 2017 Aeg: Gomez, 2003; Zonneveld et al., 2013;

Aydin and Uzar, 2014; Aydin et al., 2015

Lev: Gomez, 2003; Rubino et al., 2017

Mar: Aydin and Uzar, 2014; Balkis et al., 2016

Alb, Lig: Gómez, 2003

Bal: Gómez, 2003; Zonneveld et al., 2013 (as V.

calvum); Satta et al, 2013

Tyr: Montresor et al., 1998; Gómez, 2003;

Zonneveld et al., 2013 (as V. calvum);

Fertouna-Bellakhal et al., 2014

Ion: Rubino et al., 2002; Zonneveld et al., 2013

(as V. calvum); Ferraro et al., 2017

Aeg: Zonneveld et al., 2013 (as V. calvum);

Aydin and Uzar, 2014; Aydin et al., 2015

Lev: Gomez, 2003; Aydin and Uzar, 2014;

Rubino et al., 2017

Mar: Balkis et al., 2016

Adr: Gomez, 2003; Zonneveld et al., 2013 (as V. calvum)

Alb, Alg, Bal, Adr: Gómez, 2003; Zonneveld et al., 2013 (as T. applanatum)

Tyr: Montresor et al., 1998; Gómez, 2003;

Zonneveld et al., 2013 (as T. applanatum)

Ion: Rubino et al., 2002; Gomez, 2003;

Zonneveld et al., 2013 (as T. applanatum);

Ferraro et al., 2017 (as T. applanatum)

Aeg: Gomez, 2003; Zonneveld et al., 2013 (as

T. applanatum); Aydin et al., 2015

Mar: Aydin and Uzar, 2014; Balkis et al., 2016

Lev: Gomez, 2003

All: Gómez, 2003

Bal: Satta et al., 2013, 2014

Tyr: Montresor et al., 1998; Fertouna-Bellakhal et al., 2014

Ion: Rubino et al., 2002; Ferraro et al., 2017

Aeg: Aydin and Uzar, 2014; Aydin et al., 2015

Lev: Rubino et al., 2017

Mar: Aydin and Uzar, 2014; Balkis et al., 2016
All: Zonneveld et al., 2013 (as X.

xanthum)

NW: Rubino et al., 2000;

Sangiorgi and Donders, 2004

NE: Rubino et al., 2000

mW: Rubino et al., 2000

all: Gómez, 2003; Zonneveld et al., 2013

SE: Viličić et al., 2002

NE: Viličić et al., 2002; Cabrini et al., 2012

NW: Rubino et al., 2000; Sangiorgi and Donders, 2004 mW: Rubino et al., 2000

all: Gómez, 2003; Zonneveld et al., 2013

SE: Saracino and Rubino, 2006b

NE: Nichetto et al., 1995;

Cabrini et al., 2012

NW: Rubino et al., 2000; Sangiorgi and Donders, 2004 mW: Rubino et al., 2000 SW: Caroppo et al., 1999; Rubino et al., 2002

all: Gómez, 2003; Zonneveld et al., 2013

SE: Saracino and Rubino, 2006b NW: Sangiorgi and Donders, 2004

mW: Rubino et al, 2000

SW: Rubino et al., 2002

All: Gómez, 2003

SE: Viličić et al., 2002; Rubino et al., 2009; Drakulović et al., 2012

mE: Viličić et al., 2002

NE: Nichetto et al., 1995;

Rubino et al., 2000; Viličić

et al., 2002; Cabrini et al., 2012;

Rogelja et al., 2018

NW: Rubino et al., 2000;

Bastianini et al., 2016

mW: Caroppo, 2000; Rubino

et al., 2000

SW: Caroppo et al., 1999;

Rubino et al., 2002, 2009

(continued on next page) 
Table 5 (continued)

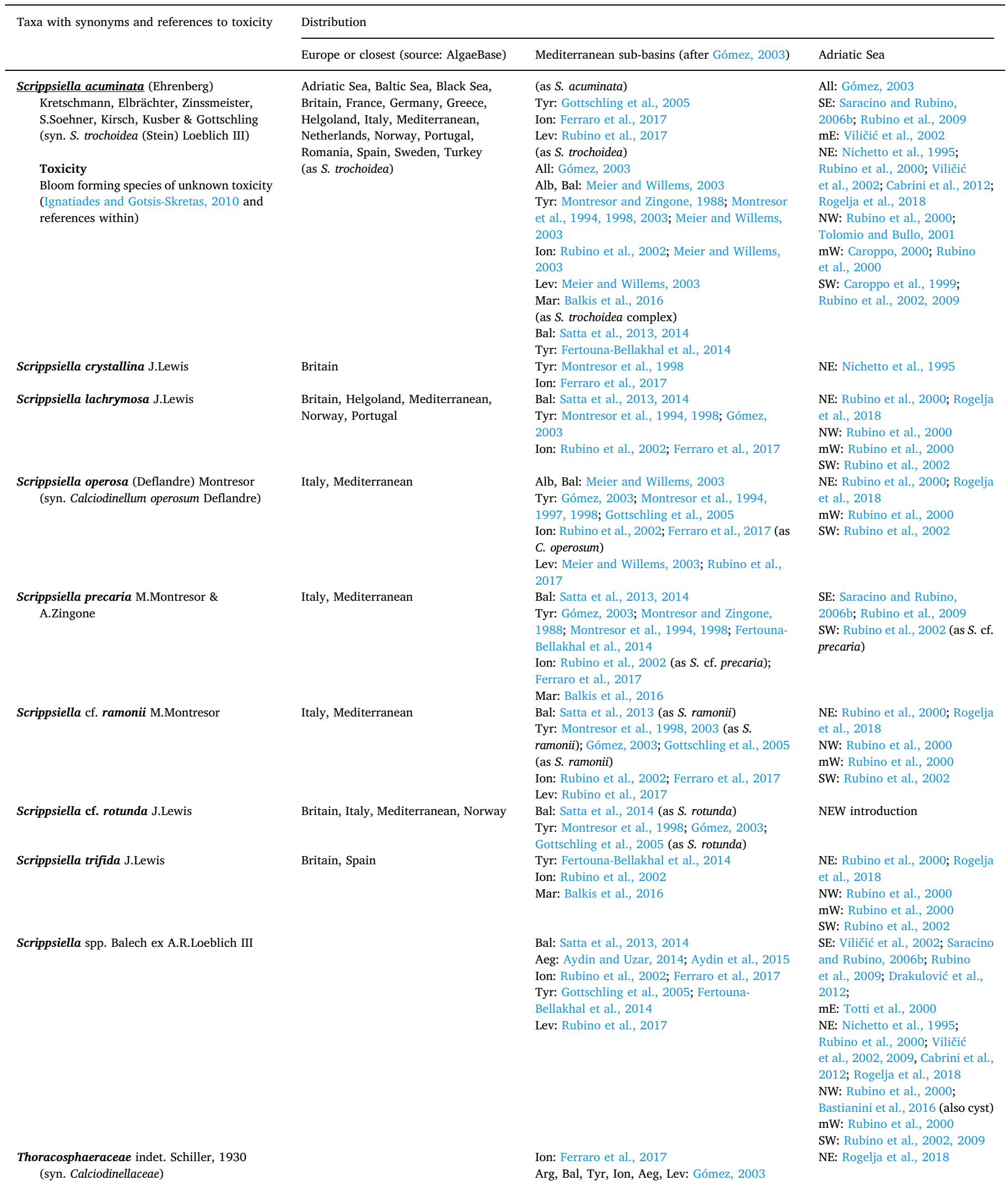


Table 6

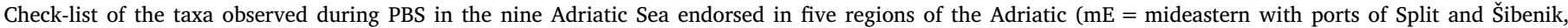

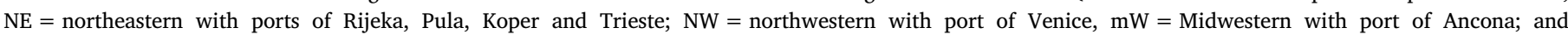

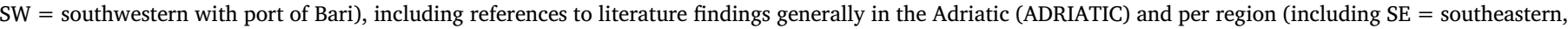
$\mathrm{mE}, \mathrm{NE}, \mathrm{NW}, \mathrm{mW}$ and SW) based on information given in Tables 4 and 5. First reports in bold, for entire Adriatic on grey.

\begin{tabular}{|c|c|c|c|c|c|c|c|c|c|c|c|c|}
\hline & \multirow{2}{*}{$\begin{array}{c}\text { ADRIATIC } \\
\text { Lit. }\end{array}$} & \multicolumn{2}{|r|}{ NW } & \multicolumn{2}{|r|}{ NE } & \multicolumn{2}{|c|}{ MW } & \multicolumn{2}{|r|}{ ME } & \multicolumn{2}{|r|}{ SW } & \multirow{2}{*}{$\begin{array}{l}\text { SE } \\
\text { Lit. }\end{array}$} \\
\hline & & Lit. & Ports & Lit. & Ports & Lit. & Ports & Lit. & Ports & Lit. & Ports & \\
\hline Alexandrium affine & & & & & PU RI & & & & & & & \\
\hline Alexandrium margaleffii & & & & & TS & & & & & & & \\
\hline Alexandrium minutum/affine/tamutum & $x$ & $x$ & VE & $x$ & TS RI PU KO & $x$ & AN & $x$ & ST SI & $x$ & $\mathrm{BI}$ & \\
\hline Alexandrium spp. & & $x$ & & $x$ & TS PO RI & $x$ & & $x$ & ST & $x$ & & $x$ \\
\hline Biecheleria spp. & & $x$ & & & RI PU KO & & & & & & & \\
\hline Cochlodinium spp. & & & & $x$ & & & & & ST SI & $x$ & & $x$ \\
\hline Diplopsalis group & $x$ & & & $x$ & TS & $x$ & & $x$ & ST SI & $x$ & $\mathrm{BI}$ & $x$ \\
\hline Gonyaulax scrippsae & $x$ & $x$ & & $x$ & TS KO RI & $x$ & & & ST SI & $x$ & & \\
\hline Gonyaulax spinifera & $x$ & $x$ & VE & $x$ & TS KO & $x$ & & $x$ & ST SI & $x$ & $\mathrm{BI}$ & $x$ \\
\hline Pentapharsodinium dalei & $x$ & $x$ & & $x$ & TS KO & & & & & $x$ & & \\
\hline Pentapharsodinium tyrrhenicum & $x$ & & & $x$ & TS KO & & & & & $x$ & & $x$ \\
\hline Polykrikos hartmanii & & & & & PU RI & & & & & & BI & \\
\hline Polykrikos spp. & $x$ & $x$ & & $x$ & TS KO & $x$ & & $x$ & & $x$ & & $x$ \\
\hline Preperidinium meunieri & & & & & $\mathbf{R I}$ & & & & ST & & BI & \\
\hline Protoceratium reticulatum & $x$ & $x$ & & $x$ & TS KO & & & & ST & $x$ & & \\
\hline Protoperidinium cf. avellana & $x$ & $x$ & & & TS & & & & & & BI & \\
\hline Protoperidinium claudicans & & $x$ & & $x$ & RI & & & & & & BI & \\
\hline Protoperidinium compressum & & $x$ & & $x$ & PU RI & $x$ & & & ST & $x$ & $\mathrm{BI}$ & \\
\hline Protoperidinium conicoides & $x$ & $x$ & & & & & & & ST & & BI & \\
\hline Scrippsiella acuminata & $x$ & $x$ & & $x$ & TS KO & $x$ & & $x$ & ST SI & $x$ & $\mathrm{BI}$ & $x$ \\
\hline Scrippsiella crystallina & & & & $x$ & TS KO & & & & & & & \\
\hline Scrippsiella lachrymosa & & $x$ & & $x$ & TS KO & $x$ & & & & $x$ & $\mathrm{BI}$ & \\
\hline Scrippsiella operosa & & & & $x$ & TS KO & $x$ & & & & $x$ & & \\
\hline Scrippsiella precaria & & & & & TS KO & & & & & $x$ & & $x$ \\
\hline Scrippsiella cf. ramonii & & $x$ & & $x$ & TS & $x$ & & & & $x$ & & \\
\hline Scrippsiella cf. rotunda & & & & & TS KO & & & & & & & \\
\hline Scrippsiella trifida & & $x$ & & $x$ & TS & $x$ & & & & $x$ & & \\
\hline Scrippsiella spp. & & $x$ & VE & $x$ & TS KO RI & $x$ & AN & $x$ & & $x$ & $\mathrm{BI}$ & $x$ \\
\hline Thoracosphaeraceae indet. & & & & $x$ & TS & & & & & & & \\
\hline round brown cysts & & & VE & & TS KO RI & & AN & & SI & & $\mathrm{BI}$ & \\
\hline small round cysts & & & & & & & & & ST SI & & & \\
\hline N.I. cysts & & & & & TS KO PU RI & & & & ST & & $\mathrm{BI}$ & \\
\hline
\end{tabular}




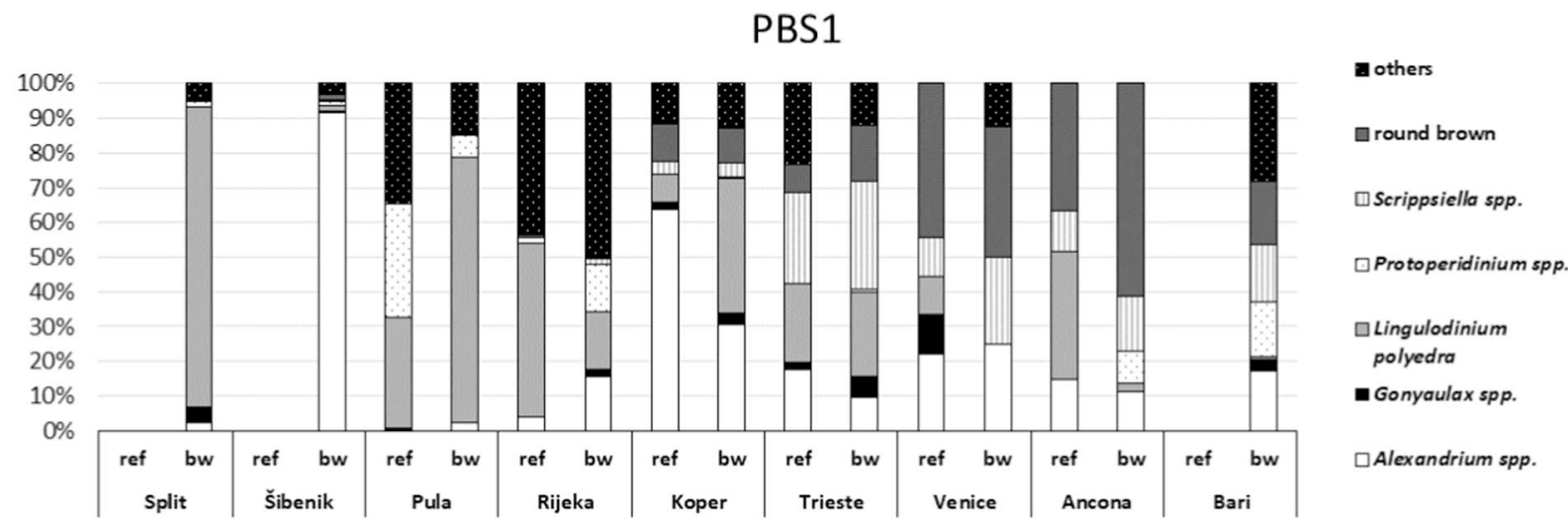

PBS2

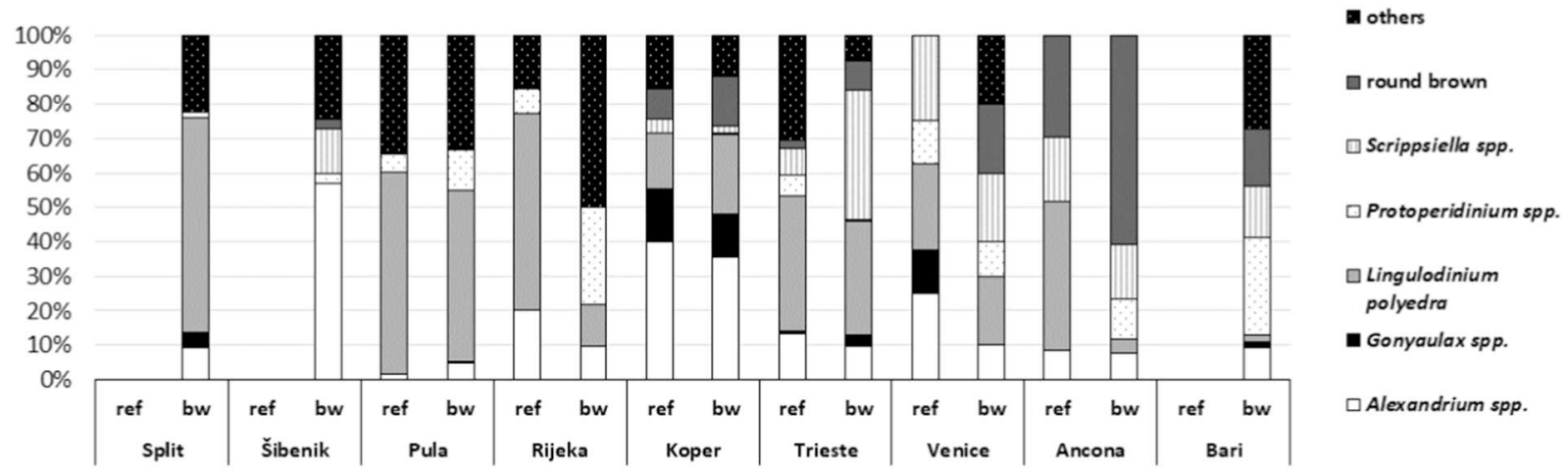

Fig. 2. Contribution in each port of the dinoflagellates cysts groups at sampling sites impacted by ballast water (bw) and controls (ref) in the first and second Port Baseline Survey (PBS1 and PBS2). All the samples of PBS1 go from April to July, except for Rijeka and Pula (December 2014) and Šibenik (November 2013); samples of PBS2 go from October to December except for Pula and Rijeka (April 2015) and Trieste (March 2015).

Koper - On average, a slight increase of cysts abundance and decrease of cyst diversity was observed between the first PBS sampling in June 2014 and the second one in November 2014. Control location values were above the averages of cysts abundance and diversity in the port. June control location was characterised by undetermined Alexandrium cysts (50\%), A. minutum/affine/tamutum group (13\%) and undetermined cysts (20\%), while at influenced locations, lower contribution of undetermined Alexandrium cysts (24\%), L. polyedra (39\%) and same contribution of undetermined cysts were observed. In November, in entire port there was a dominance of undetermined Alexandrium cysts $(31 \%)$, L. polyedra $(21 \%)$, and generally undetermined cysts (23\%) and specifically of the genus Gonyaulax (13\%). During this PBS, Protoperidinium divaricatum was found only in port of Koper, at influenced locations on both sampling dates.

Trieste - When two spring PBS samplings are compared (May 2014 and March 2015), a noticeable decrease of cysts abundance and diversity increase can note between the two. At the control location in May 2014, cysts abundance and diversity were considerably lower than at impacted ones, while in March 2015, values at all investigated locations were rather similar. At one influenced station, TSbw1 in March 2015, 20 taxa were observed, which is maximum of the entire PBS, while this port was generally characterised by highest diversity. Control location in May 2014 was dominated by L. polyedra (40\%) and undetermined cysts (30\%), while at impacted locations, besides L. polyedra (33\%) and undetermined cysts (15\%), undetermined cysts of Scrippsiella (24\%) and Alexandrium genus (8\%) were reported. In March 2015, cysts assemblage was dominated by Alexandrium and Scrippsiella genuses, while the most abundant species were L. polyedra (24\%), Scrippsiella acuminata (7\%), undetermined cysts of Scrippsiella genus (17\%), and other undetermined cysts (25\%). During this PBS, several species were found only in port of Trieste: Alexandrium insuetum,
Alexandrium margaleffii and Protoperidinium cf. avellana (only in March), Scrippsiella ramonii and Scrippsiella trifida (in May and March), only at impacted locations. Interestingly, undetermined cysts from Thoracosphaeraceae were reported at impacted location in May 2014 and at control location in March 2015.

Venice - During the two PBS samplings, in May and October 2014, the lowest abundances of entire PBS were reported for this port (average of $6 \mathrm{~g}^{-1} \mathrm{DW}$ ). Cyst diversity was among lowest observed, in total 7 taxa over both samplings. Higher contribution in May was reported for undetermined cysts of genus Scrippsiella (18\%), cysts identified as group A. minutum/affine/tamutum (24\%) and unidentified cysts (41\%), and in October, first two remained with contributions of 22 and 17\%, respectively, with L. polyedra (22\%). No species were observed solely in this port.

Ancona - During the two PBS samplings, in July and October 2014, cysts abundance was close to minimal values of entire PBS and diversity was PBS lowest, in total 5 taxa over both samplings. Interestingly, during both samplings, the most dominant was L. polyedra (40\%), with unidentified cysts (33\%) at control location, while at impacted ones, contribution of L. polyedra was negligible (3\%), whereas unidentified cysts remained present in noticeably elevated contribution (61\%). No species were observed solely in this port.

Bari - The two samplings, performed in May and November 2014, revealed that in comparison with other ports, rather low cyst abundance and high cyst diversity, characterise this one. In this port no control location was investigated. At influenced locations in May dominated $A$. minutum/affine/tamutum group (11\%) and S. acuminata (10\%), with large amount of undetermined and round brown cysts (42\%). In November, undetermined cysts were observed in decreased amount (28\%), with dominant cysts of genus Protoperidinium (20\%) and Preperidinium meunieri $(10 \%)$. No species were observed solely in this port. 
Table 7

PRIMER SIMPER procedure calculations of average similarity (SIM.) and average dissimilarity (DISS.) (both expressed as \%) based on original abundance dataset modified to include original referent values and averaged values from all impacted sampling stations based on $\sqrt{ } V$-transformed values and Bray-Curtis similarities. Seasons 1 and 2 stand generally for samplings performed in two various months (for details on sampling months see Table 2).

\begin{tabular}{|c|c|c|c|c|c|c|c|c|c|c|c|c|}
\hline & ST & SI & RI & PU & KO & TS & $\mathrm{VE}$ & AN & BI & MIN & MAX & AVE \\
\hline SIM. all stations & 48.76 & 57.50 & 58.09 & 72.67 & 74.82 & 61.58 & 65.35 & 79.47 & 84.16 & 48.76 & 84.16 & 66.42 \\
\hline SIM. referent stations & n.a. & n.a. & 60.08 & 71.91 & 77.59 & 56.83 & 75.00 & 95.98 & n.a. & 56.83 & 95.98 & 72.90 \\
\hline SIM. impacted stations & $48.76^{\mathrm{b}}$ & $57.50^{\mathrm{b}}$ & 60.14 & 79.76 & 82.27 & 72.68 & 76.47 & 96.53 & $84.16^{\mathrm{b}}$ & 48.76 & 96.53 & 73.14 \\
\hline DISS. referent-impacted & n.a. & n.a. & 42.92 & 28.91 & 27.73 & 40.01 & 39.85 & 28.92 & n.a. & 27.73 & 42.92 & 34.72 \\
\hline SIM. season 1 & n.a. & n.a. & 53.53 & 71.38 & 69.73 & 62.19 & 65.05 & 70.86 & n.a. & 53.53 & 71.38 & 65.46 \\
\hline SIM. season 2 & n.a. & n.a. & 63.62 & 67.42 & 75.53 & 55.60 & 65.46 & 71.30 & n.a. & 55.60 & 75.53 & 66.49 \\
\hline DISS. season 1-2 & $51.24^{\mathrm{a}}$ & 42.50 & 42.15 & 25.69 & 24.08 & 37.08 & 34.61 & 16.33 & 15.84 & 15.84 & 51.24 & 32.17 \\
\hline
\end{tabular}

${ }^{\text {a }}$ In port Split, data was collected over three different months and only an average is given here.

${ }^{b}$ In ports Bari, Sibenik and Split no samplings were performed at referent locations, therefore similarities based on data at all stations equal similarities from impacted ones.

\subsection{Cyst assemblage comparison}

Our similarity analysis based on specific port dataset, including all data, and between control-impacted stations, as well as seasonal data (Table 7) revealed that overall cyst assemblage in port of Bari is most consistent (highest similarity per port, 84.16\%) and between two seasons (lowest dissimilarity, 15.84\%). In port of Ancona assemblage is most consistent at impacted and referent stations (highest similarity, around 96\%). Cyst assemblage of the highest variability was found in port of Split (lowest similarity, $48.76 \%$ ), due to high seasonal variability (highest dissimilarity, 51.24\%). The most prominent difference between cyst communities between impacted and control stations was

Table 8

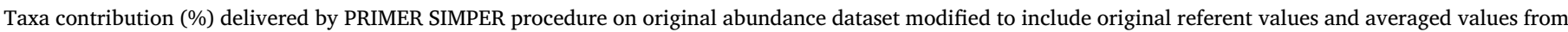

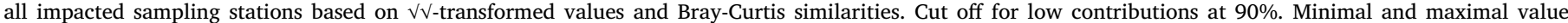
calculated on basis $\geq 2$ ports, and median $\geq 3$ ports.

\begin{tabular}{|c|c|c|c|c|c|c|c|c|c|c|c|c|c|}
\hline Taxa PORT & ST & SI & RI & PU & KO & TS & $\mathrm{VE}$ & AN & BI & MIN & MAX & AVE & MED \\
\hline Alexandrium affine & & & & & & & & & & - & - & - & - \\
\hline Alexandrium insuetum & & & & & & & & & & - & - & - & - \\
\hline Alexandrium margalefii & & & & & & & & & & - & - & - & - \\
\hline Alexandrium minutum/affine/tamutum & 18.15 & 27.11 & 17.47 & & 9.98 & 8.34 & 28.20 & 21.18 & 7.89 & 7.89 & 28.20 & 17.29 & 17.81 \\
\hline Alexandrium tamarense/catenella & & & & & & 5.67 & & & 6.91 & 5.67 & 6.91 & 6.29 & - \\
\hline Alexandrium spp. & & & & & 14.80 & 9.46 & & & & 9.46 & 14.80 & 12.13 & - \\
\hline Biecheleria spp. & & & 18.97 & 8.57 & 6.68 & & & & & 6.68 & 18.97 & 11.41 & 8.57 \\
\hline Cochlodinium spp. & & & & & & & & & & - & - & - & - \\
\hline Diplopsalis group & 5.43 & 13.55 & & & & & & & & 5.43 & 13.55 & 9.49 & - \\
\hline Gonyaulax scrippsae & 17.72 & & & & & & & & & - & - & 17.72 & - \\
\hline Gonyaulax spinifera & & & & & & 4.93 & 4.57 & & 5.54 & 4.57 & 5.54 & 5.01 & - \\
\hline Gonyaulax spp. & & & & & 8.48 & 3.34 & & & & 3.34 & 8.48 & 5.91 & - \\
\hline Gymnodinium impudicum & 12.47 & & & & & & & & & - & - & 12.47 & - \\
\hline Gymnodinium spp. & & & 14.10 & 6.06 & & & & & & 6.06 & 14.10 & 10.08 & - \\
\hline Lingulodinium polyedra & 32.11 & & 25.24 & 25.46 & 12.83 & 14.37 & 13.62 & 18.48 & 4.83 & 4.83 & 32.11 & 18.37 & 16.43 \\
\hline Pentapharsodinium dalei & & & & & 2.89 & 2.34 & & & & 2.34 & 2.89 & 2.62 & - \\
\hline Pentapharsodinium tyrrhenicum & & & & & & & & & & - & - & - & - \\
\hline Polykrikos hartmannii & & & & 17.32 & & & & & & - & - & 17.32 & - \\
\hline Polykrikos spp. & & & & & & 2.80 & & & & - & - & 2.80 & - \\
\hline Preperidinium meunieri & & & & & & & & & 6.50 & - & - & 6.50 & - \\
\hline Protoceratium reticulatum & 4.55 & & & & & & & & & - & - & 4.55 & - \\
\hline Protoperidinium cf. avellana & & & & & & & & & & - & - & - & - \\
\hline Protoperidinium claudicans & & & & & & & & & 5.47 & - & - & 5.47 & - \\
\hline Protoperidinium compressum & & & & & & & & & 5.13 & - & - & 5.13 & - \\
\hline Protoperidinium conicoides & & & & & & & & & & - & - & - & - \\
\hline Protoperidinium conicum & & & & & & 2.51 & & & & - & - & 2.51 & - \\
\hline Protoperidinium divaricatum & & & & & & & & & & - & - & - & - \\
\hline Protoperidinium leonis & & & & & & & & & 6.21 & - & - & 6.21 & - \\
\hline Protoperidinium oblongum & & & & & & & & & & - & - & - & - \\
\hline Protoperidinium pentagonum & & & & & & & & & & - & - & - & - \\
\hline Protoperidinium spp. & & 13.55 & 15.17 & 15.41 & & & & & 8.44 & 8.44 & 15.41 & 13.14 & 14.36 \\
\hline Scrippsiella acuminata & & 16.12 & & & 2.82 & 4.25 & & & 7.89 & 2.82 & 16.12 & 7.77 & 6.07 \\
\hline Scrippsiella crystallina & & & & & & & & & & - & - & - & - \\
\hline Scrippsiella lachrymosa & & & & & & & & & 7.54 & - & - & 7.54 & - \\
\hline Scrippsiella operosa & & & & & 2.90 & & & & & - & - & 2.90 & - \\
\hline Scrippsiella precaria & & & & & & & & & & - & - & - & - \\
\hline Scrippsiella cf. ramonii & & & & & & & & & & - & - & - & - \\
\hline Scrippsiella cf. rotunda & & & & & & & & & & - & - & - & - \\
\hline Scrippsiella trifida & & & & & & & & & & - & - & - & - \\
\hline Scrippsiella spp. & & & & & 6.29 & 11.23 & 29.62 & 23.77 & & 6.29 & 29.62 & 17.73 & 17.50 \\
\hline Thoracosphaeraceae indet. & & & & & & & & & & - & - & - & - \\
\hline Round brown & & 13.55 & & & 11.49 & 9.23 & 15.73 & 32.86 & 10.02 & 9.23 & 32.86 & 15.48 & 12.52 \\
\hline Small round cysts & & 16.12 & & & & & & & & - & - & 16.12 & - \\
\hline N.I. cysts & & & & 18.50 & 11.44 & 12.5 & & & 9.41 & 9.41 & 18.50 & 12.96 & 12.96 \\
\hline
\end{tabular}




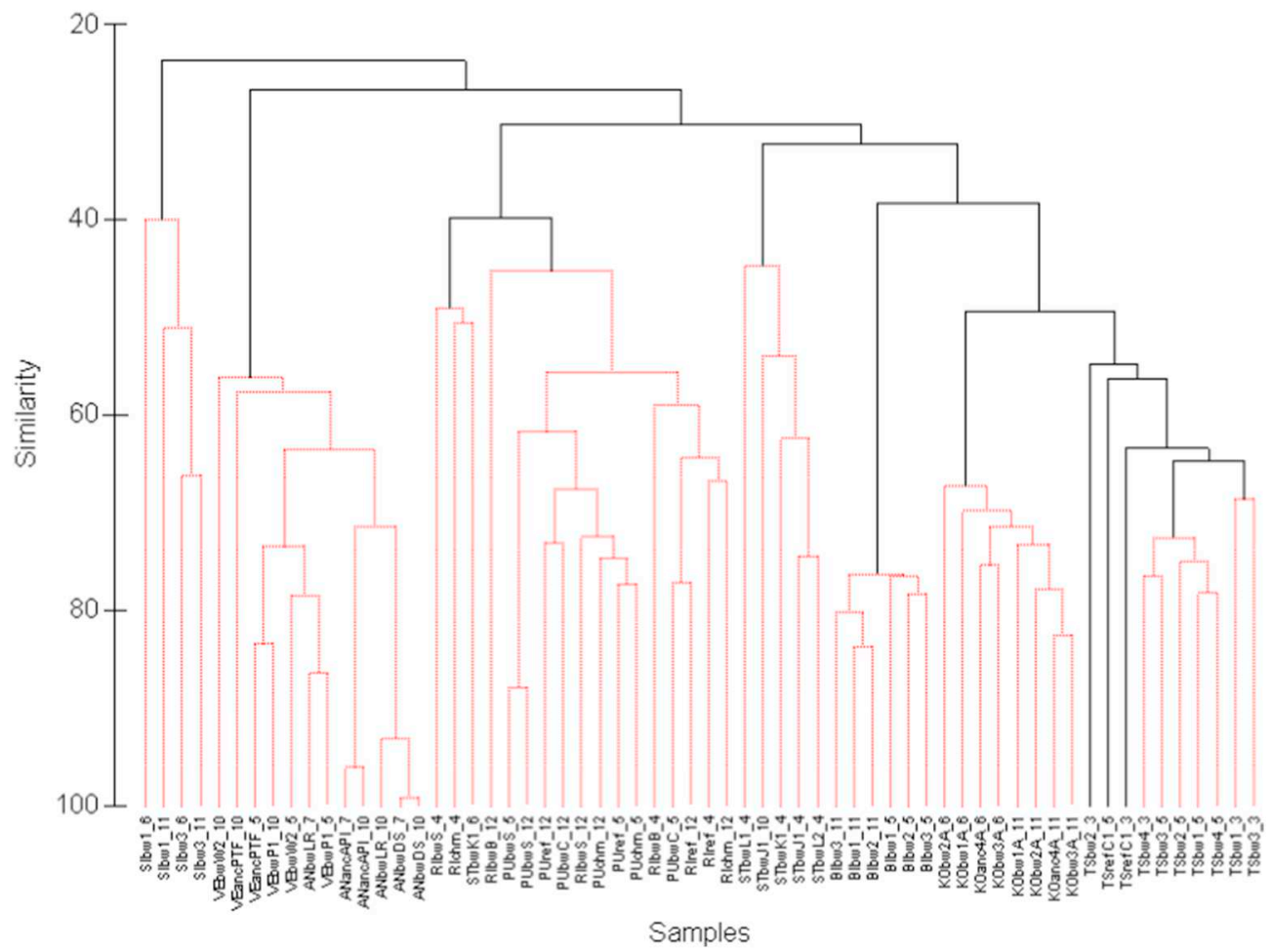

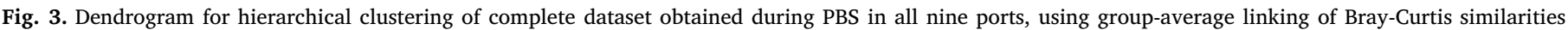
calculated on $\sqrt{ } \sqrt{ }$-transformed abundance data. Dotted branches indicate significant relationships resulting from SIMPROF test.

detected in port of Rijeka (dissimilarity $42.92 \%$ ).

In general, the dissimilarity between control and impacted stations, as well as between seasonal data, is very low (32-35\%, indicating communities to be rather uniform inside one particular port, regardless of observed taxa number in respective port (as low as in port of Ancona, or as high as in port of Trieste).

Taxa composition among the ports did not exhibit significant differences, indeed the most abundant species appear to be largely shared by all the ports (Table 8). Three most dominant taxa, based on highest taxa contribution in each port $(>18 \%)$ and observation frequency $(\geq 4$ ports), were L. polyedra (in 8 ports, with average contribution of $18 \%$ ), A. minutum/affine/tamutum (in 8 ports, with $17 \%$ ) and unidentified Scrippsiella species (in 4 ports, with 18\%). Interestingly, the subsequent three taxa which followed by high contribution (16-17\%), were of scarce frequency, one port only, Gonyaulax scrippsae in Split, Polykrikos hartmannii in Pula and small round cysts in Šibenik. In contrast, taxa composition changed significantly among ports as confirmed by ANOSIM, Global $R=0.909$, significance level $<0.001$. The differences among ports are mainly due to: 1) marked dissimilarities in biomass and frequency of a pool of taxa common to all ports, and 2) the presence of specific taxa that were associated, albeit to different degrees, with individual port.

Port coherency investigation performed by Cluster (Fig. 3) and MDS ordination (Fig. 4) with the integral cysts dataset delivered outcome of spatial aggregation among the ports: Trieste - Koper, than with Bari and Split at lower similarity, followed by Rijeka - Pula, than Venice - Ancona, whereas Šibenik showed least similarity with the rest of the ports. The MDS ordination showed, above all, a significant difference between samples collected in different ports. Further similarity investigation by SIMPER procedure (Table 9) indicated lowest dissimilarity between

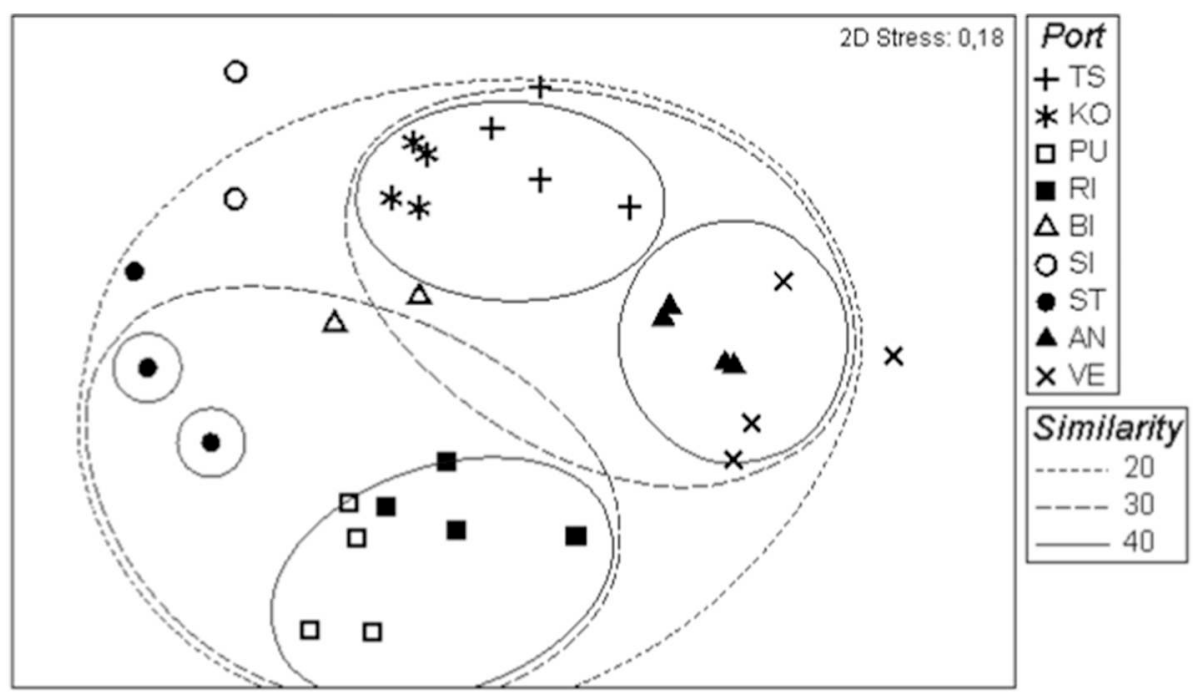

Fig. 4. Two-dimensional MDS configuration of original abundance dataset modified to include original referent values and averaged values from all impacted sampling stations based on $\mathrm{V} V$-transformed values and Bray-Curtis similarities with superimposed clusters performed at complete dataset as presented at Fig. 3 (similarity levels 20, 30 and 40). 
Table 9

PRIMER SIMPER procedure calculations of average dissimilarity (\%) between the ports, based on original abundance dataset modified to include original referent values and averaged values from all impacted sampling stations based on $\checkmark \mathrm{V}$-transformed values and Bray-Curtis similarities. Dissimilarities above and under range of first standard deviation $(66.6 \pm 10.0 \%)$ were marked in bold, and highest/lowest are within square.

\begin{tabular}{llllllllll}
\hline & ST & SI & RI & PU & KO & TS & VE & AN & BI \\
\hline ST & & & & & & & & & \\
SI & 61,8 & & & & & & & & \\
RI & 65,2 & 72,8 & & & & & & & \\
PU & 66,4 & $\mathbf{7 9 , 6}$ & $\mathbf{4 2 , 2}$ & & & & & & \\
KO & 64,0 & 68,2 & 63,9 & 64,8 & & & & & \\
TS & 73,3 & 72,7 & 68,8 & 73,8 & $\mathbf{4 8 , 5}$ & & & & \\
VE & $\mathbf{8 4 , 1}$ & $\mathbf{7 8 , 6}$ & 67,3 & $\mathbf{8 1 , 2}$ & $\mathbf{7 8 , 8}$ & 66,2 & & & \\
AN & $\mathbf{7 8 , 1}$ & 67,7 & 62,4 & 76,0 & 65,9 & 60,5 & $\mathbf{3 8 , 4}$ & & \\
BI & 68,7 & $\mathbf{5 6 , 7}$ & 62,4 & 61,4 & 61,5 & 57,4 & $\mathbf{7 2 , 8}$ & 65,5 & \\
\hline
\end{tabular}

\section{Table 10}

Main taxa accountable for dissimilarity between the ports (highest contribution) obtained by PRIMER SIMPER procedure calculations based on original abundance dataset modified to include original referent values and averaged values from all impacted sampling stations based on $\sqrt{ } \mathrm{V}$-transformed values and Bray-Curtis similarities. Alexandrium minutum/affine/tamutum (Amat). Alexandrium spp. (Alex). Biecheleria spp. (Biec). Gonyaulax scrippsae (Gscr). Lingulodinium polyedra (Lpol). Polykrikos hartmannii (Phar). Protoperidinium spp. (Prot). N.I. cysts (N.I.) and round brown (R.B.).

\begin{tabular}{llllllllll}
\hline & ST & SI & RI & PU & KO & TS & VE & AN & BI \\
\hline ST & & & & & & & & \\
SI & Lpol & & & & & & & \\
RI & Lpol & Amat & & & & & & \\
PU & Gscr & Amat & Phar & & & & & \\
KO & Alex & Amat & Alex & Alex & & & & \\
TS & Lpol & Amat & Biec & Phar & Alex & & & \\
VE & Lpol & Amat & Biec & Lpol & Alex & N.I. & & \\
AN & Lpol & Amat & R.B. & N.I. & Alex & N.I. & R.B. & \\
BI & Lpol & Amat & R.B. & Lpol & Alex & Prot & N.I. & N.I. \\
\hline
\end{tabular}

Ancona and Venice (38.4\%), low between Pula and Rijeka, Trieste and Koper, while the high dissimilarity was among pairs, Venice-Koper, Venice-Pula, Venice-Šibenik, Ancona-Split, Pula-Šibenik, and the highest between Venice and Split (84.1\%). In 27 out of 36 pairs that nine investigated ports form, there were only four taxa attributed as a major foundation of dissimilarity in the pair as delivered by SIMPER (Table 10); Alexandrium cysts determined only to genus level (8 pairs), L. polyedra (8 pairs), A. minutum/affine/tamutum group (6 pairs) and generally unidentified cysts (5 pairs).

\section{Discussion}

In addition to regular production of dinoflagellate cysts during sexual reproduction phase, encystment can be triggered in response to unfavourable environmental conditions (Taylor, 1987; Garcés et al., 2002). Accordingly, in addition to being introduced in ship tanks during ballast intake, cysts formation may be triggered by unfavourable conditions for vegetative stages during voyages (Casas-Monroy et al., 2013). During an 18 months investigation of phytoplankton contained in ballast tanks of 19 cargo vessels calling in Taranto port, a total of 48 phytoplanktonic species in the water and 51 different morphotypes of resting stages in the sediments were recorded, representing 17 dinoflagellate genera (Saracino and Rubino, 2006a). Due to difficulties encountered in collecting, the results were reported to be, almost certainly, underestimated. Another study focused on variability between tanks proved that species diversity and their relative abundance showed significant variability, when a total of 21 dinoflagellate species were identified from 31 distinctive cyst morphotypes in four ballast water tanks of a single ship (Hamer et al., 2000). Reports show that the resting stages produced by potentially toxic dinoflagellate species of the genus Alexandrium were present in $40 \%$ of the sediment samples from the Taranto port (Saracino and Rubino, 2006a), and occurrence of potentially toxic species, Alexandrium tamarense and other unidentified Alexandrium cyst morphotypes detected in three of the four investigated tanks in another study (Hamer et al., 2000), confirm and substantiate the concern of the inadvertent transport of the potentially toxic dinoflagellate species (Hallegraeff and Bolch, 1991, 1992).

As the ballast water tanks are usually cleaned only when the ship is in dry dock, within each separate ballast tank of an individual ship, a mixture of sediments from several different ports typically accumulates, with high potential for development of different assemblages of dinoflagellate resting stages originated from several years of vessel operations (Hamer et al., 2000). Their extreme resilience enables cysts to endure ship transport for several years (Rigby and Hallegraeff, 1994). Although sediments accumulate at the bottom of ballast tanks, their resuspension during de-ballasting enables any amount of its content to be discharged (Villac et al., 2001). Ports represent a logical place to initiate biological surveys as the most likely area for new marine introduction by BW (Awad et al., 2014). Furthermore, confined areas in coastal areas, such as ports, were reported to host high densities and diversities of cysts assemblages (Rubino et al., 2002).

The PBS conducted as one of research activities of the BALMAS project, included investigation of dinoflagellate resting stages in nine ports along the Adriatic, including 26 sampling stations impacted by BW and 6 control locations. Our comparison of dinoflagellate cysts assemblages based on sampling location types, i.e., between the ones that are potentially under direct impact of BW and those without any or under indirect impacts (control), pointed out that essentially there are no observable differences. The highest dissimilarity between cyst assemblage at impacted and control station was obtained in port of Rijeka. However, the rationale behind this result is hardly a straightforward one. The topography of the port itself seems to have low impact on potential cysts spread, based on all dissimilarities calculations between impacted and control sites of the investigated ports. Locations of control sites, which were outside ports in coastal area of the "open" type ports, like Trieste, Koper and Pula, or outside ports in open coastal zone of the "closed" type ports, like Venice (naturally enclosed inside a lagoon), Ancona and Rijeka (artificially enclosed ports), did not reflect in any diversities of the cysts assemblages respective to sampling site type. Although choice of control and impacted sampling sites was based on current understanding of the circulation pattern of the respective port, we are inclined to conclude that choice of control sites have to be further afield, in an area of high settling rate, and limited connection to the port by circulation pattern. As detailed investigations of the ports seem to be rare (e.g., Mali et al., 2017), this issue might remain challenging in the close future.

Irrespective of sampling location type, our investigation of cysts assemblages among the ports revealed seasonal variability to be most pronounced in port of Split, high in Rijeka and Šibenik, low in Ancona, Koper and Pula, and least evident in Bari. However, differences seem to be restricted to quantitative aspect, as qualitatively, cyst assemblage remained rather constant inside each specific port. Evidently lower cyst abundance, respective to other ports, was observed in ports of Ancona and Venice, which is in contrast to the general observations that in secluded areas (e.g., ports), limited hydrodynamics favours accumulation of sinking cysts within the sediments (e.g., Satta et al., 2010, 2013) but we have to consider that Venice (Umgiesser et al., 2004) and Ancona (Fabi et al., 2016) present peculiar higher hydrodinamism. This finding highlights the complexity of factors inducing encystment and their settlement and accumulation. Actually, hydrographic and sedentary processes, such as currents and cysts sinking rates, appear to be also species selective, complicating further understanding of cysts settlement process (Matsuoka et al., 2003). 
Dinoflagellate cyst assemblages were predominated by heterotrophic organic Peridiniales at the western side of the Adriatic, comprising ports of Bari, Ancona and Venice, and autothropic/mixotrophic Gonyaulacales at the eastern. Other autotrophs, namely calcareus Peridiniales, were codominant in all western ports, and one eastern, port of Trieste. However, often referred to as a widely distributed and present in high relative abundances, Scrippsiella acuminata (e.g., Persson et al., 2000; Satta et al., 2013; Wang et al., 2007) was restricted to five ports, Trieste, Koper, Split, Šibenik and Bari, while only in last two it was observed in high relative abundances $(\sim 10-13 \%)$. Interestingly, Gymnodiniales occasionally contributed with considerable abundances to the cyst assemblage. Specifically, with Polykrikos hartmannii in port of Pula (in May and December), with unidentified Gymnodinium species in ports of Rijeka (especially in April), Split (in June) and Venice (especially in October), and in Šibenik, Polykrikos (in November).

General separation to the western and eastern side of the Adriatic, regarding cysts assemblage composition, and partially abundance, potentially might be related to events triggered by bora, pronounced at the eastern coast (e.g., Alpers et al., 2009). Bora is dry strong north to northeastern wind blowing along the eastern Adriatic coast usually during cold part of the year; episodes last on average for $40 \mathrm{~h}$, being strongest for about 12 (Orlić et al., 1994; Cushman-Roisin et al., 2001). Bora episodes can induce strong evaporation and consequently impact the circulation pattern of the Adriatic (e.g., Lyons et al., 2007; Supić et al., 2012); inducing mixing of the water column, usually in the eastern (Wang et al., 2007), and during severely intense episodes, also in the western Adriatic (Boldrin et al., 2009). Efficient mixing of the water column at the eastern coast during bora might potentially favour intense cysts settlement at the eastern side of the Adriatic, resulting in distinct abundance gradient. Role of surface heat losses in phytoplankton dynamics and introducing species from sediment into water column was already discussed by Kraus and Supić (2015), whereas here we suggest the opposite, the settlement of phytoplankton cysts from the water column into the sediment. High contribution of heterotrophic taxa might also be related to observed eutrophic conditions in coastal waters (Thorsen and Dale, 1997; Dale et al., 1999; Matsuoka, 1999; Dale, 2001, 2009), and to the heavy impact of the prevailing sewage and industrial pollution (Matsuoka, 1999). Additionally, trophicity of the taxa might be accountable, as distribution of heterotrophic dinoflagellates is partially controlled by prey availability (diatoms and small flagellates) (Matsuoka, 1999), while those of phototrophic depends on light and dissolved nutrients availability (Dale, 2001). There is also a possibility that ports at the western side are more impacted by BW, in which heterotrophic taxa were found predominant over autotrophic, consequential to light deprivation within ballast water tanks, facilitating higher resilience and survival rate of the heterotrophs (CasasMonroy et al., 2013).

One species of large distribution over the Adriatic coast, which was observed in all investigated ports, was autotrophic Lingulodinium polyedra (Gonyaucales). Indeed, this ubiquitous species is widely distributed, from temperate zones in Northern to subtropical in Southern hemisphere, over rather wide temperature range (above $10^{\circ} \mathrm{C}$ in summer and $0{ }^{\circ} \mathrm{C}$ in winter) and in a broad salinity range (Zonneveld et al., 2013), with noted preference for lower salinity, and relatively high nutrient concentration (e.g., Zonneveld et al., 2010). However, the relative contribution and abundances of $L$. polyedra in our ports ranged considerably. Hence, it was the quantitative aspect of $L$. polyedra which was accountable as one of four main sources of dissimilarity identified among studied ports. We believe the explanation is probably port specific as distribution range of $L$. polyedra cysts potentially can be inflicted by a variety of external factors inducing $L$. polyedra encystment, such as turbulence (Figueroa and Bravo, 2005). Some of the highest relative abundances were reported in the vicinity of the active upwelling cells and near river mouths, and seasonal production upon development of stratified upper waters following turbulence (Zonneveld et al., 2013). Furthermore, high $L$. polyedra abundances are considered to be related to eutrophication (Dale and Fjellså, 1994; Dale et al., 1999), relatively warmer sea surface temperatures (Thorsen and Dale, 1997), lower salinity (Morzadec-Kerfourn, 1977), or even almost entirely limited to estuarine systems (Wall et al., 1977), whereas one survey suggests association to estuarine areas subjected to eutrophication or land-runoff (Persson et al., 2000). Other taxa accountable for dissimilarity identified among studied ports were Alexandrium minutum/affine/tamutum group, undetermined taxa of the genus Alexandrium, and generally undetermined dinoflagellate morphotypes.

Despite shortcomings of a relatively low sample counts to substantiate reliable conclusions regarding BW impacts in different ports, general insight regarding cysts distribution is rather attainable. Our results are consistent with observations of a study conducted in the Gulf of Trieste (Nichetto et al., 1995) where taxa detected in port and coastal area have only a fair number of taxa shared among both areas (9), with several restricted to port (6), and a higher number exclusively to the coastal area (18). Namely, during this study numerous taxa were not detected in port/ $\mathrm{s}$ which are endorsed in previously reported region of observation (Tables 5 and 6). For example, Scrippsiella lachrymosa was reported for several regions of the Adriatic, NW and $\mathrm{mW}$ (Rubino et al., 2000), SW (Rubino et al., 2002) and NE (Rubino et al., 2000; Rogelja et al., 2018). However, during this study it was observed only in ports of Trieste and Koper (NW region) and Bari (SW region), thus confirming that spreading taxa over entire region, including port areas, is not necessarily a process which is certain to occur. This is also documented by rather high dissimilarity of cyst communities between ports of Venice and Trieste or Koper, all endorsed in the Northern Adriatic, a part of the Adriatic often characterised by a closed circulatory pattern separating this one from the other parts of the Adriatic. On the other hand, spreading between the two ports in close proximity seems to have occurred, as observed for Trieste-Koper, PulaRijeka or even for Venice-Ancona, although this was not observed in the case of ports of Šibenik and Split, which are also two rather proximate ports. For example, Biecheleria spp. was reported in NW region (Bastianini et al., 2016), but was undetected in port of Venice during this study, whereas Protoperidinium conicum was reported in $\mathrm{mE}$ region (Viličić et al., 2002), but was observed only in port of Split during this study (and not in port of Šibenik).

Spreading of dinoflagellates over coastal areas, and across the Adriatic by natural pattern, by general circulatory system and/or large gyres connecting western and eastern part of Adriatic, seems to be rather limited. Nevertheless, three taxa were observed in a new region during this study, whereto the spreading might have occurred by general anticyclonic currents system: Scrippsiella precaria spread from SE (Saracino and Rubino, 2006b; Rubino et al., 2009) up the eastern coast to NE (observed in port/s of Trieste and Koper), and Protoperidinium claudicans down the western coast, from NW (Sangiorgi and Donders, 2004; Bastianini et al., 2016) down to SW (observed in port of Bari). Protoperidinium compressum, observed in port of Split might have spread by large gyres eastwards from mW (Rubino et al., 2000) and/or SW (Rubino et al., 2002, 2009) and then up the eastern coast by general anticyclonic system. We want to point out that their spread might have occurred also by BW, from intra-Adriatic marine traffic, or from BW originating from outside the Adriatic. For more detailed insight into Adriatic circulatory pattern related to potential spreading of species as opposed to BW transfer refer to Kraus and co-authors (2019b).

In this study we confirmed the presence of taxa already described in literature for some Adriatic regions (Tables 5 and 6). However, these taxa were reported also as generally present in the Adriatic, without specifying any particular region (Gómez, 2003; Zonneveld et al., 2013). Now, we point out their observation in a region/s unreported until now: A. tamarense/catenella group, Gonyaulax scrippsae, Protoceratium reticulatum, and Protoperidinium pentagonum were observed in $\mathrm{mE}$ during this study, and Protoperidinium conicoides and Protoperidinium leonis in $\mathrm{mE}$ and SW.

Finally, for the remaining six taxa no previous reports of observation were attainable, thus are assumed as NIS in the Adriatic. Specifically, these taxa were observed in six ports, as follows: in port of Bari Polykrikos hartmanii and Preperidinium meunieri, in port of Koper Scrippsiella cf. 
rotunda, in port of Rijeka Alexandrium affine, Polykrikos hartmanii, and Preperidinium meunieri, in port of Pula Alexandrium affine and Polykrikos hartmanii, in port of Trieste Alexandrium insuetum, Alexandrium margaleffii, and Scrippsiella cf. rotunda, and in port of Split Preperidinium meunieri.

As already stated, our investigation indicated that considerable diversity exists among the regions along the Adriatic coast. We observed two taxa which are included in the provisional list of harmful aquatic organisms in the Adriatic, representing a constituent part of the Early Warning System for Non Indigenous Species for the Adriatic (Magaletti et al., 2018), so granting any intra-Adriatic exemptions from BW management should be taken carefully taking into account also this aspect. For example, A. tamarense/catenella group is not yet introduced to ports of Venice or Ancona, and P. reticulatum to ports of Pula, Rijeka and Šibenik considering also taxa distribution findings of vegetative forms in seawater column reported by Mozetič et al. (this issue). As concluded in the Baltic Sea case study, in order to ensure data reliability, in addition to PBS, regular monitoring programs should be undertaken during the exemption period as findings of new species influence the results of risk assessment for exemptions from BW management (David et al., 2013). As presented results of this study indicate, introduction of NIS and toxic taxa have occurred over the last years, and the potential threat of BW to Adriatic represents an issue yet to be addressed. International Maritime Organization (IMO) Ballast Water Management (BWM) Convention for the Control and Management of Ship's Ballast Water and Sediments (IMO, 2004) has entered into force on 8 September 2017. BWM Convention sets requirements to prevent organism transfers, thus enhancing prospects of improved protection of marine environment from any harmful impacts of shipping in the near future. One of the aims this study is helping scenariospecific risk assessments that help reducing risk of invasion in susceptible areas. Other approaches should be followed: BW treatment (physical, chemical or simply by introducing mid-way ballast exchange) is one of the main efforts to be gradually introduced by the new convention (David et al., 2015).

Observation of nine toxic dinoflagellate taxa (of variable toxicity, ranging from unknown/low to toxin producers), might impose an issue in extra-Adriatic traffic by meeting the criteria of issuing a warning to ships related to the risk assessment criteria of the Guidelines for Risk Assessment under Regulation A-4 of the BWM Convention (G7) (MEPC, 2007), i.e., the warning aimed at minimizing the uptake of BW which could be harmful for the recipient port or area (David et al., 2013, 2015). It has long ago been suggested that risk assessment systems might support identification of vessels likely to contain NIS or particularly undesirable species (Hayes, 1998). However, a need for accurate and readily available ships ballast log records in combination with information of the spatial and temporal distribution of target species in port areas around the world was already claimed in the past (Hamer et al., 2000).

However, some promising technical developments might enable improvements in addressing the issue of potential threats by BW. Namely, the accurate determination of dinoflagellate vegetative forms and resting stages based on microscopic examination requiring extensive taxonomic expertise, with intrinsic limitations of the method, lead to development of molecular identification techniques (Bolch, 2001; Godhe et al., 2002; Erdner et al., 2010; Penna et al., 2007). Although crosscheck between the microscopic and molecular methods is indispensable before independent application of the latter, one of the most beneficial and obvious advantages is ultimately faster identification on a high resolution of species-level (John et al., 2014). Following development of single cyst/cell-based method successfully applied at autotrophs (Bolch, 2001), especially belonging to genera of Alexandrium and Gymnodinium (Penna et al., 2008; Bolch, 2001; Godhe et al., 2002; Lin et al., 2006), recently new dinoflagellate-specific primers have been designed to increase the PCR efficiency for heterotrophs (Gao et al., 2017). The use of these faster molecular methods was tested also on limited number of ports during BALMAS PBS (Perini et al., this issue) with promising results to facilitate more efficient monitoring efforts in the future. Importance of this issue is highlighted even more as considerable amount of cyst remained unidentified during processing samples in this study, providing additional source of potential NIS, or even harmful and toxic taxa.

\section{Acknowledgements}

This publication has been produced with the financial assistance of the IPA Adriatic Cross-Border Cooperation Programme - strategic project Ballast Water Management System for Adriatic Sea Protection (BALMAS). The contents of this publication are the sole responsibility of authors and can under no circumstances be regarded as reflecting the position of the IPA Adriatic Cross-Border Cooperation Programme Authorities. This work was additionally funded by The National Foundation for Science, Higher Education and Technological Development of the Republic of Croatia, project Ecological response of northern Adriatic to climatic changes and anthropogenic impact (EcoRENA). The authors are grateful to crew of research vessel Vila Velebita, M/B Litus, dr. Andrej Jaklin and Darko Ferenčević and Italian Coast Guard diver team for sediment sampling by diving routine, and dr. Alfred Beran, Denis Škalic, Ivan Balković and Jernej Uhan for assistance in sediment processing.

\section{References}

Alpers, W., Ivanov, A., Horstmann, J., 2009. Observations of bora events over the Adriatic Sea and Black Sea by spaceborne synthetic aperture radar. Mon. Weather Rev. 137, 1150-1161.

Anderson, D.M., Chisholm, S.W., Watras, C.J., 1983. Importance of life cycle events in the population dynamics of Gonyaulax tamarensis. Mar. Biol. 76 (2), 179-189.

Anderson, D.M., Fukuyo, Y., Matsuoka, K., 2003. Cyst methodologies. In: Hallegraeff, G.M., Anderson, D.M., Cembella, A. (Eds.), Manual on Harmful Marine Microalgae. Monographs on Oceanographic Methodology 11. Unesco, Paris, pp. 165-189.

Anderson, D.M., Stock, C.A., Keafer, B.A., Nelson, A.B., Thompson, B., McGillicuddy, D.J., Keller, M., Thompson, B., Matrai, P.A., Martin, J., 2005. Alexandrium fundyense cyst dynamics in the Gulf of Maine. Deep-Sea Res. II 52 (19), 2522-2542.

Anderson, D.M., Cembella, A.D., Hallegraeff, G.M., 2012. Progress in understanding harmful algal blooms (HABs): paradigm shifts and new technologies for research, monitoring and management. Annu. Rev. Mar. Sci. 4, 143-176. https://doi.org/10. 1146/annurev-marine-120308-081121.

Anglès, S., Jordi, A., Garcés, E., Basterretxea, G., Palanques, A., 2010. Alexandrium minutum resting cyst distribution dynamics in a confined site. Deep-Sea Res. II 57 (3), 210-221.

Awad, A., Haag, F., Anil, A.C., Abdulla, A., 2014. GEF-UNDP-IMO GloBallast Partnerships Programme, IOI, CSIR-NIO and IUCN. Guidance on Port Biological Baseline Surveys. GEF-UNDP-IMO GloBallast Partnerships, London, UK. GloBallast Monograph No. 22.

Aydin, H., Uzar, S., 2014. Distribution and abundance of modern dinoflagellate cysts from Marmara, Aegean and Eastern Seas of Turkey. J. Environ. Biol. 35, 413-419.

Aydin, H., Yürür, E.E., Uzar, S., Küçüksezgin, F., 2015. Impact of industrial pollution on recent dinoflagellate cysts in Izmir Bay (Eastern Aegean). Mar. Pollut. Bull. 94, 144-152. https://doi.org/10.1016/j.marpolbul.2015.02.038.

Baldwin, R.P., 1992. Cargo vessel ballast water as a vector for the spread of toxic phytoplankton species to New Zealand. J. R. Soc. N. Z. 22, 229-242.

Balkis, N., Balci, M., Giannakourou, A., Venetsanopoulou, A., Mudie, P., 2016. Dinoflagellate resting cysts in recent marine sediments from the Gulf of Gemlik (Marmara Sea, Turkey) and seasonal harmful algal blooms. Phycologia 55 (2), 187-209.

Bastianini, M., Totti, C., Penna, A., De Lazzari, A., Montresor, M., 2016. Dinoflagellate cysts production in the north-western Adriatic Sea. Mediterr. Mar. Sci. 17 (3), 751-765.

Bates, H.A., Kostriken, R., Rapoport, A., 1978. The occurrence of saxitoxin and other toxins in various dinoflagellates. Toxicon 16 (6), 595-601.

Belmonte, G., Castello, P., Piccinni, M.R., Quarta, S., Rubino, F., Geraci, S., Boero, F., 1995. Resting stages in marine sediments off the Italian coasts. In: Eleftheriou, A., Ansell, A.D., Smith, C.J. (Eds.), Biology and Ecology of Shallow Coastal Waters. Olsen and Olsen, Fredensborg, pp. 53-58.

Berman, J., Harris, L., Lambert, W., Buttrick, M., Dufresne, M., 1992. Recent invasions of the Gulf of Maine: three contrasting ecological histories. Conserv. Biol. 6, 435-442.

Bernardi Aubry, F., Berton, A., Bastianini, M., Socal, G., Acri, F., 2004. Phytoplankton succession in a coastal area of the NW Adriatic, over a 10-year sampling period (1990-1999). Cont. Shelf Res. 24 (1), 97-115.

Blanco, J., 1986. Separacion de quistes de dinoflagelados en gradiente de densidad. Bol. Inst. Esp. Oceanogr. 3, 81-84.

Boero, F., Belmonte, G., Fanelli, G., Piraino, S., Rubino, F., 1996. The continuity of living matter and the discontinuities of its constituents: do plankton and benthos really exist? Trends Ecol. Evol. 11, 177-180.

Bolch, C.J.S., 1997. The use of sodium polytungstate for the separation and concentration of living dinoflagellate cysts from marine sediments. Phycologia 36 (6), 472-478. 
Bolch, C.J.S., 2001. PCR protocols for genetic identification of dinoflagellates directly from single cysts and plankton cells. Phycologia 40 (2), 162-167. https://doi.org/10. 2216/i0031-8884-40-2-162.1.

Boldrin, A., Carniel, S., Giani, M., Marini, M., Bernardi Aubry, F., Campanelli, A., Grilli, F., Russo, A., 2009. Effects of bora wind on physical and biogeochemical properties of stratified waters in the northern Adriatic. J. Geophys. Res. 114, C08S92. https://doi. org/10.1029/2008JC004837.

Bravo, I., Garcés, E., Diogène, J., Fraga, S., Sampedro, N., Figueroa, R.I., 2006. Resting cysts of the toxigenic dinoflagellate genus Alexandrium in recent sediments from the Western Mediterranean coast, including the first description of cysts of $A$. kutnerae and A. peruvianum. Eur. J. Phycol. 41 (3), 293-302.

Bringue, M., Pospelova, V., Calvert, S.E., Enkin, R.J., Lacourse, T., Ivanochko, T., 2016. High resolution dinoflagellate cyst record of environmental change in Effingham Inlet (British Columbia, Canada) over the last millennium. Palaeogeogr. Palaeoclimatol. Palaeoecol. 441 (4), 787-810.

Bruno, M., Gucci, P.M.B., Pierdominici, E., Ioppolo, A., Volterra, L., 1990. Presence of saxitoxin in toxic extracts from Gonyaulax polyedra. Toxicon 28 (9), 1113-1116.

Buttermore, R.E., Turner, E., Morrice, M.G., 1994. The introduced northern Pacific seastar Asterias amurensis in Tasmania. Queensland Mus. Mem. 36, 21-25.

Cabrini, M., Fornasaro, D., Cossarini, G., Lipizer, M., Virgilio, D., 2012. Phytoplankton temporal changes in a coastal northern Adriatic site during the last 25 years. Estuar. Coast. Shelf Sci. 115, 113-124.

Cabrini, M., Cerino, F., de Olazabal, A., Di Poi, E., Fabbro, C., Fornasaro, D., Goruppi, A., Flander-Putrle, V., Francé, J., Gollasch, S., Hure, M., Lipej, L., Lučić, D., Magaletti, E., Mozetič, P., Tinta, T., Tornambè, A., Turk, V., Uhan, J., David, M., 2019. Potential transfer of aquatic organisms via ballast water with a particular focus on harmful and non-indigenous species: A survey from Adriatic ports. (This issue).

Camacho, F.G., Rodríguez, J.G., Mirón, A.S., García, M.C., Belarbi, E.H., Chisti, Y., Grima, E.M., 2007. Biotechnological significance of toxic marine dinoflagellates. Biotechnol. Adv. 25 (2), 176-194.

Candel, M.S., Radi, T., de Vernal, A., Bujalesky, G., 2012. Distribution of dinoflagellate cysts and other aquatic palynomorphs in surface sediments from the Beagle Channel, Southern Argentina. Mar. Micropaleontol. 96, 1-12.

Carlton, J.T., 1985. Transoceanic and interoceanic dispersal of coastal marine organisms: the biology of ballast water. Oceanogr. Mar. Biol. Annu. Rev. 23, 313-374.

Carlton, J.T., 1987. Patterns of transoceanic marine biological invasions in the Pacific Ocean. Bull. Mar. Sci. 41 (2), 452-465.

Carlton, J.T., 1989. Man's role in changing the face of the ocean: biological invasions and implications for conservation of near-shore environments. Conserv. Biol. 3 (3), 265-273.

Carlton, J.T., 1999. Molluscan invasions in marine communities. Malacologia 41, 439-454.

Carlton, J.T., Geller, J.B., 1993. Ecological roulette: the global transport and invasion of nonindigenous marine organisms. Science 261, 78-82.

Caroppo, C., 2000. The contribution of picophytoplankton to community structure in a Mediterranean brackish environment. J. Plankton Res. 22 (2), 381-397.

Caroppo, C., Fiocca, A., Sammarco, P., Magazzù, G., 1999. Seasonal variations of nutrients and phytoplankton in the coastal SW Adriatic Sea (1995-1997). Bot. Mar. 42 (4), 389-400.

Casas-Monroy, O., Roy, S., Rochon, A., 2013. Dinoflagellate cysts in ballast sediments differences between Canada's east coast, west coast and the Great Lakes. Aquat. Conserv. Mar. Freshwat. Ecosyst. 23, 254-276.

Chapman, J.W., Carlton, J.T., 1991. A test of criteria for introduced species: the global invasion by the isopod Synidotea laevidorsalis (Miers, 1881). J. Crustac. Biol. 11, 386-400.

Chu, K.H., Tam, P.F., Fung, C.H., Chen, Q.C., 1997. A biological survey of ballast water in container ships entering Hong Kong. Hydrobiologia 352, 201-206.

Clarke, K.R., Warwick, R.M., 2001. Change in Marine Communities: An Approach to Statistical Analysis and Interpretation, Second ed. PRIMER-E, Plymouth (172 pp).

Coutts, A.D.M., Kirrily, M.M., Chad, L.H., 2003. Ships' sea-chests: an overlooked transfer mechanism for non-indigenous marine species? Mar. Pollut. Bull. 46, 1504-1515.

Cushman-Roisin, B., Gačić, M., Poulain, P.-M., Artegiani, A. (Eds.), 2001. Physical Oceanography of the Adriatic Sea. Kluwer Academic Publishers, Dordrecht, The Netherlands, pp. 304.

Dale, B., 1983. Dinoflagellate resting cysts: "benthic plankton". In: Fryxell, G.A. (Ed.), Survival Strategies of the Algae. Cambridge University Press, pp. 69-136.

Dale, B., 2001. Marine dinoflagellate cysts as indicators of eutrophication and industrial pollution: a discussion. Sci. Total Environ. 264, 235-240.

Dale, B., 2009. Eutrophication signals in the sedimentary record of dinoflagellate cysts in coastal waters. J. Sea Res. 61, 103-113.

Dale, B., Dale, A.L., 2002. Dinoflagellate contributions to the deep sea. In: Ocean Biocoenosis Series, No. 5 Woods Hole Oceanographic Institution, Wood Hole, MA, USA.

Dale, B., Fjellså, A., 1994. Dinoflagellate cysts as paleoproductivity indicators: state of the art, potential and limits. In: Zahn, R., Pedersen, T.F., Kaminski, M.A., Labeyrie, L. (Eds.), Carbon Cycling in the Glacial Ocean: Constraints on the Ocean's Role in Global Change. NATO ASI Series I 17. Springer-Verlag, Berlin Heidelberg, pp. 521-537.

Dale, B., Thorsen, T.A., Fjellså, A., 1999. Dinoflagellate cysts as indicators of cultura eutrophication in the Oslofjord, Norway. Estuar. Coast. Shelf Sci. 48, 371-382.

David, M., Gollasch, S., Cabrini, M., Perkovič, M., Bošnjak, D., Virgilio, D., 2007. Results from the first ballast water sampling study in the Mediterranean Sea-the Port of Koper study. Mar. Pollut. Bull. 54 (1), 53-65.

David, M., Gollasch, S., Leppäkoski, E., 2013. Risk assessment for exemptions from ballast water management - the Baltic Sea case study. Mar. Pollut. Bull. 75, 205-217.

David, M., Gollasch, S., Leppäkoski, E., Hewitt, C., 2015. Risk assessment in ballast water management. In: David, M., Gollasch, S. (Eds.), Global Maritime Transport and
Ballast Water Management-Issues and Solutions. Springer Science + Business Media, Dordrecht Heidelberg New York, London. https://doi.org/10.1007/978-94-017 9367-4.

Drakulović, D., Pestorić, B., Cvijan, M., Krivokapić, S., Vuksanović, N., 2012. Distribution of phytoplankton community in Kotor Bay (south-eastern Adriatic Sea). Open Life Sci. 7 (3), 470-486.

Ellegaard, M., 2000. Variations in dinoflagellate cyst morphology under conditions of changing salinity during the last 2000 years in the Limfjord, Denmark. Rev. Palaeobot. Palynol. 109 (1), 65-81. https://doi.org/10.1016/S0034-6667(99) 00045-7.

Ellegaard, M., Daugbjerg, N., Rochon, A., Lewis, J., Harding, I., 2003. Morphological and LSU rDNA sequence variation with the Gonyaulax spinifera-Spiniferites group (Dinophyceae) and proposal of $G$. elongate comb. nov. and G. membranacea comb. nov. Phycologia 42, 151-164. https://doi.org/10.2216/i0031-8884-42-2-151.1.

Erdner, D.L., Percy, L., Keafer, B., Lewis, J., Anderson, D.M., 2010. A quantitative realtime PCR assay for the identification and enumeration of Alexandrium cysts in marine sediments. Deep-Sea Res. II 57 (3), 279-287. https://doi.org/10.1016/j.dsr2.2009. 09.006 .

Evagelopoulos, A., Nikolaidis, G., 1996. Morphology of Protoperidinium compressum (Peridiniales, Dinophyceae) in the North Aegean Sea, Greece. Nova Hedwigia 63, 301-307.

Fabi, G., Ferrarin, C., Grilli, F., Penna, P., Spagnolo, A., 2016. Modellizzazione della diffusione del materiale sedimentario risospeso durante i lavori di escavo e di sversamento in mare aperto - rapporto finale. In: Rapporto tecnico per l'Autorità Portuale di Ancona, (51 pp).

Faust, M.A., 1990. Cysts of Prorocentrum marinum (Dinophyceae) in floating detritus at Twin Cays, Belize man-grove habitats. In: Graneh, E., Sundstrom, B., Edler, L. Anderson, D.M. (Eds.), Toxic Marine Phytoplankton. Proc Fourth Int Conterence Toxic Marine Phytoplankton, 26-30 June 1989, Lund, Sweden. Elsevier Pub, New York, pp. 138-143.

Faust, M.A., 1993. Sexuality in a toxic dinoflagellate, Prorocentrum lima. In: Smayda, T.J., Shimizu, Y. (Eds.), Toxic Phytoplankton Blooms in the Sea. Proc 5th Int Conference on Toxic Marine Phytoplankton, 28 Oct-1 Nov 1991, Newport, USA. Elsevier SCI Pub, New York, pp. 121-126.

Ferraro, L., Rubino, F., Belmonte, M., Da Prato, S., Greco, M., Frontalini, F., 2017. A multidisciplinary approach to study confined marine basins: the holobenthic and merobenthic assemblages in the Mar Piccolo of Taranto (Ionian Sea, Mediterranean). Mar. Biodivers. 47 (3), 887-911. https://doi.org/10.1007/s12526-016-0523-0.

Fertouna-Bellakhal, M., Dhib, A., Béjaoui, B., Turki, S., Aleya, L., 2014. Driving factors behind the distribution of dinocyst composition and abundance in surface sediments in a western Mediterranean coastal lagoon: report from a high resolution mapping study. Mar. Pollut. Bull. 84 (1-2), 347-362. https://doi.org/10.1016/j.marpolbul 2014.04.041.

Figueroa, R.I., Bravo, I, 2005. Sexual reproduction and two different encystment strategies of Lingulodinium polyedrum (Dinophyceae) in culture. J. Phycol. 41, 370-379.

Gao, Y., Fang, H., Dong, Y., Li, H., Pu, C., Zhan, A., 2017. An improved method for the molecular identification of single dinoflagellate cysts. PeerJ 5, e3224. https://doi. org $/ 10.7717 /$ peerj.3224.

Garcés, E., Maso, M., Camp, J., 1999. A recurrent and localized dinoflagellate bloom in Mediterranean beach. J. Plankton Res. 21, 2373-2391.

Garcés, E., Masó, M., Camp, J., 2002. Role of temporary cysts in the population dynamics of Alexandrium taylori (Dinophyceae). J. Plankton Res. 24, 681-686.

Giannakourou, A., Orlova, T.Y., Assimakopoulou, G., Pagou, K., 2005. Dinoflagellate cysts in recent marine sediments from Thermaikos Gulf, Greece: effects of resuspension events on vertical cyst distribution. Cont. Shelf Res. 25 (19), 2585-2596.

Godhe, A., Rehnstam-Holm, A.F., Karunasagar, I., Karunasagar, I., 2002. PCR detection of dinoflagellate cysts in field sediment samples from tropic and temperate environments. Harmful Algae 1, 361-373. https://doi.org/10.1016/S1568-9883(02) 00053-7.

Gollasch, S., 1998. Removal of Barriers to the Effective Implementation of Ballast Water Control and Management Measures in Developing Countries. pp. 197 For GEF/IMO/ UNDP.

Gollasch, S., 2002. The importance of ship hull fouling as a vector of species introductions into the North Sea. Biofouling 18, 105-121.

Gollasch, S., Lenz, J., Dammer, M., Andres, H.G., 2000a. Survival of tropical ballast wate organisms during a cruise from the Indian Ocean to the North Sea. J. Plankton Res. 22, 923-937.

Gollasch, S., Rosenthal, H., Botnen, H., Hamer, J., Laing, I., Leppakoski, E., Macdonald, E., Minchin, D., Nauke, M., Olenin, S., Utting, S., Voigt, M., Wallentinus, I., 2000b. Fluctuations of zooplankton taxa in ballast water during short-term and long-term ocean-going voyages. Int. Rev. Hydrobiol. 85, 597-608.

Gollasch, S., MacDonald, E., Belson, S., Botnen, H., Christensen, J.T., Hamer, J.P., Houvenaghel, G., Jelmert, A., Lucas, I., Masson, D., McCollin, T., Olenin, S., Persson, A., Wallentinus, I., Wetsteyn, L.P.M.J., Wittling, T., 2002. Life in ballast tanks. In: Leppakoski, E., Gollasch, S., Olenin, S. (Eds.), Invasive Aquatic Species of Europe: Distribution, Impacts and Management. Kluwer Academic Publishers, Dordrecht, pp. 217-231.

Gómez, F., 2003. Checklist of Mediterranean free-living dinoflagellates. Bot. Mar. 46, 215-242.

Gottschling, M., Knop, R., Plötner, J., Kirsch, M., Willems, H., Keupp, H., 2005. A molecular phylogeny of Scrippsiella sensu lato (Calciodinellaceae, Dinophyta) with in terpretations on morphology and distribution. Eur. J. Phycol. 40, 207-220.

Graneli, E., Codd, A., Dale, B., Lipiatou, E., Maestrini, S.Y., Rosenthal, H., 1998. Harmful algal blooms in European marine and brackish waters. In: Proceedings of EUROHAB, Science Initiative, EUR 18592, 5-7 November, Kalmar, Sweden.

Gu, H.F., Zeng, N., Xie, Z.X., Wang, D.Z., Wang, W.G., Yang, W.D., 2013. Morphology, 
phylogeny, and toxicity of Atama complex (Dinophyceae) from the Chukchi Sea. Polar Biol. 36 (3), 427-436. https://doi.org/10.1007/s00300-012-1273-5.

Hallegraeff, G.M., 1993. A review of harmful algal blooms and their apparent global increase. Phycologia 32, 79-99.

Hallegraeff, G.M., 2010. Ocean climate change, phytoplankton community responses, and harmful algal blooms: a formidable predictive challenge. J. Phycol. 46, 220-235.

Hallegraeff, G.M., Bolch, C.J., 1991. Transport of toxic dinoflagellate cysts via ships' ballast water. Mar. Pollut. Bull. 22, 27-30.

Hallegraeff, G.M., Bolch, C.J., 1992. Transport of diatom and dinoflagellate resting spores in ships' ballast water: implications for plankton biogeography and aquaculture. J. Plankton Res. 14, 1067-1084.

Hallegraeff, G.M., Bolch, C.J., Bryan, J., Koerbin, B., 1990. Microalgal spores in ships' ballast water: a danger to aquaculture. In: Graneli, E. (Ed.), Toxic Marine Phytoplankton. Elsevier, New York, pp. 475-480.

Hamer, J.P., McCollin, T.A., Lucas, I.A.N., 2000. Dinofagellate cysts in ballast tank sediments: between tank variability. Mar. Pollut. Bull. 40 (9), 731-733.

Hay, C., Handley, S., Dogdshun, T., Taylor, M., Gibbs, W., 1997. Cawthron's ballast water research programme final report 1996-97. In: Cawthron Report No. 417. Cawthron Institute, pp. 144.

Hayes, K.R., 1998. Ecological risk assessment for ballast water introductions: a suggested approach. ICES J. Mar. Sci. 55, 201-212.

Head, M.J., 1996. Modern dinoflagellate cysts and their biological affinities. In: Jansonius, J., McGregor, D.C. (Eds.), Palynology: Principles and Applications. The American Association of Stratigraphic Palynologist, Dallas, pp. 1197-1248.

Hégaret, H., Shumway, S.E., Wikfors, G.H., Pate, S., Burkholder, J.M., 2008. Potential transport of harmful algae via relocation of bivalve molluscs. Mar. Ecol. Prog. Ser. 361, 169-179.

Hewitt, C.L., Martin, R.B., 2001. Revised protocols for baseline port surveys for introduced marine species: survey design, sampling protocols and specimen handling. In: Centre for Research on Introduced Marine Pests. Technical Report No. 22. CSIRO Marine Research, Hobart (46 pp).

Honsell, G., Smayda, T.J., 1993. First report of Alexandrium minutum in northern Adriatic waters (Mediterranean Sea). In: Shimizu, Y. (Ed.), Toxic Phytoplankton Blooms in the Sea. Elsevier, Amsterdam, pp. 127-132.

Horner, R.A., Greengrove, C.L., Davies-Vollum, K.S., Gawel, J.E., Postel, J.R., Cox, A.M., 2011. Spatial distribution of benthic cysts of Alexandrium catenella in surface sediments of Puget Sound, Washington, USA. Harmful Algae 11, 96-105.

Howarth, R.S., 1981. Presence and implication of foreign organisms in ship ballast waters discharged into the Great Lakes. In: Report to the Environmental Protection Service, Environment Canada, Ottawa. Bio-Environmental Services Ltd., Georgetown, Ontario (97 pp).

Hutchings, P., 1992. Ballast water introductions of exotic marine organisms into Australia: current status and management options. Mar. Pollut. Bull. 25, 196-199.

Ignatiades, L., Gotsis-Skretas, O., 2010. A review on toxic and harmful algae in Greek coastal waters (E. Mediterranean Sea). Toxins 2, 1019-1037.

IMO, 2004. International Convention for the Control and Management of Ships' Ballast Water and Sediments, 2004. International Maritime Organization, 13 February 2004. International Maritime Organization, London, UK (36 pp).

John, U., Litaker, R.W., Montresor, M., Murray, S., Brosnahan, M.L., Anderson, D.M., 2014. Formal revision of the Alexandrium tamarense species complex (dinophyceae) taxonomy: the introduction of five species with emphasis on molecular - based (rDNA) classification. Protist 165 (6), 779-804. https://doi.org/10.1016/j.protis. 2014.10.001.

Joyce, L.B., 2004. Dinoflagellate cysts in recent marine sediments from Scapa Flow, Orkney, Scotland. Bot. Mar. 47 (3), 173-183.

Joyce, L.B., Pitcher, G.C., Du Randt, A., Monteiro, P.M.S., 2005. Dinoflagellate cysts from surface sediments of Saldanha Bay, South Africa: an indication of the potential risk of harmful algal blooms. Harmful Algae 4 (2), 309-318.

Kelly, J., 1993. Ballast water and sediments as mechanisms for unwanted species introductions into Washington State. J. Shellfish Res. 12, 405-410.

Kokinos, J.P., Anderson, D.M., 1995. Morphological development of resting cysts in cultures of the marine dinoflagellate Lingulodinium polyedrum ( = L. machaerophorum). Palynology 19, 143-166.

Krajcar, V., 2003. The climatology of geostrophic currents in the northern Adriatic. Geofizika 20, 105-114.

Kraus, R., Supić, N., 2015. Sea dynamics impacts on the macroaggregates: a case study of the 1997 mucilage event in the northern Adriatic. Prog. Oceanogr. 138, 249-267.

Kraus, R., Ninčević-Gladan, Ž., Auriemma, R., Bastianini, M., Bolognini, L., Cabrini, M. Cara, M., Calić, M., Campanelli, A., Cvitković, I., Despalatović, M., Drakulović, D., Flander-Putrle, V., Grati, F., Grego, M., Grilli, F., Jaklin, A., Janeković, I., Kolitari, J., Lipej, L., Magaletti, E., Marini, M., Mavrič, B., Mikus, J., Mozetič, P., Orlando-Bonaca, M., Petović, S., Precali, R., Supić, N., Trabucco, B., Travizi, A., Žuljević, A., 2019a. Strategy of Port Baseline Survey (PBS) in the Adriatic Sea. (This issue. In press).

Kraus, R., Grilli, F., Supić, N., Janeković, I., Brailo, M., Cara, M., Cetinić, A., Campanelli, A., Cozzi, S., D'Adamo, R., Djakovac, T., Dutour-Sikirić, M., Flander Putrle, V., France, J., Joksimović, D., Klun, K., Kolitari, J., Kralj, M., Kušpilić, G., Marini, M., Matić, F., Mikus, J., Ninčević-Gladan, Ž., Pansera, M., Pećarević, M., Precali, R., Prusina, I., Relitti, F., Santucci, A., Specchiulli, A., Škalic, D., 2019b. Oceanographic Characteristics of the Adriatic Sea - Support to Secondary Spread of HAOP by Natural Dispersal. (This issue. In press).

Lin, S., Zhang, H., Hou, Y., Miranda, L., Bhattacharya, D., 2006. Development of a dinoflagellate-oriented PCR primer set leads to detection of picoplanktonic dinoflagellates from Long Island Sound. Appl. Environ. Microbiol. 72 (8), 5626-5630. https://doi.org/10.1128/AEM.00586-06.

Loader, J.I., Hawkes, A.D., Beuzenberg, V., Jensen, D.J., Cooney, J.M., Wilkins, A.L., Fitzgerald, J.M., Briggs, L.R., Miles, C.O., 2007. Convenient large-scale purification of yessotoxin from Protoceratium reticulatum culture, and isolation of a novel furanoyessotoxin. J. Agric. Food Chem. 55 (26), 11093-11100.

Lodge, D.M., 1993. Species invasions and deletions: community effects and responses to climate and habitat change. In: Kareiva, P.M., Kingsolver, J.G., Huey, R.B. (Eds.), Biotic Interactions and Global Change. Sinauer Associates, Inc., Sunderland, Massachusetts, pp. 376-387.

Lundholm, N., Ribeiro, S., Andersen, T.J., Koch, T., Godhe, A., Ekelund, F., Ellegaard, M., 2011. Buried alive-germination of up to a century-old marine protist resting stages. Phycologia 50 (6), 629-640.

Lyons, D.M., Supić, N., Smodlaka, N., 2007. Geostrophic circulation patterns in the northeastern Adriatic Sea and the effects of air-sea coupling: May-September 2003. J. Geophys. Res. 112, C03S08.

Macdonald, E.M., Davidson, R.D., 1997. Ballast Water Project, Final Report. FRS Marine Laboratory Aberdeen, pp. 83.

Macdonald, E.M., Davidson, R.D., 1998. Ballast Water Project Fisheries Research Services Report Number 3/97. FRS Marine Laboratory, Aberdeen (83 p).

MacKenzie, L., Truman, P., Satake, M., Yasumoto, T., Adamson, J., Mountfort, D., White, D., Reguera, B., 1998. Dinoflagellate blooms and associated DSP-toxicity in shellfish in New Zealand. In: Fernandez, M.L., Wyatt, T. (Eds.), Harmful Algae. Xunta de Galicia and IOC of UNESCO, pp. 74-77.

Magaletti, E., Garaventa, F., David, M., Castriota, L., Kraus, R., Luna, G.M., Silvestri, C., Forte, C., Bastianini, M., Falautano, M., Maggio, T., Rak, G., Gollasch, S., 2018. Developing and testing an early warning system for non indigenous species and ballast water management. J. Sea Res. 133, 100-111.

Mali, M., De Serio, F., Dell'Anna, M.M., Mastrorilli, P., Damiani, L., Mossa, M., 2017. Enhancing the performance of hazard indexes in assessing hot spots of harbour areas by considering hydrodynamic parameters. Ecol. Indic. 73, 38-45. https://doi.org/10. 1016/j.ecolind.2016.09.028.

Matsuoka, K., 1999. Eutrophication process recorded in dinoflagellate cyst assemblages a case of Yokohama Harbour, Tokyo Bay, Japan. Sci. Total Environ. 231, 17-35.

Matsuoka, K., Fukuyo, Y., 2000. Technical Guide for Modern Dinoflagellate Cyst Study. WESTPACHAB/WESTPAC/IOC, Japan Society of the Promotion Science, Tokyo, pp. 29.

Matsuoka, K., Joyce, L.B., Kotani, Y., Matsuyama, Y., 2003. Modern dinoflagellate cysts in hypertrophic coastal waters of Tokyo Bay, Japan. J. Plankton Res. 25 (12), 1461-1470. https://doi.org/10.1093/plankt/fbg111.

Matsuoka, K., Kawami, H., Nagai, S., Iwataki, M., Takayama, H., 2009. Re-examination of cyst-motile relationships of Polykrikos kofoidii Chatton and Polykrikos schwartzii Bütschli (Gymnodiniales, Dinophyceae). Rev. Palaeobot. Palynol. 154 (1), 79-90. https://doi.org/10.1016/j.revpalbo.2008.12.013.

Meier, K.J.S., Willems, H., 2003. Calcareous dinofagellate cysts in surface sediments from the Mediterranean Sea: distribution patterns and infuence of main environmental gradients. Mar. Micropaleontol. 48, 321-354.

MEPC, 2007. Guidelines for Risk Assessment Under Regulation A-4 of the BWM Convention (G7). Annex 2 Adopted 13 July 2007. The Marine Environment Protection Committee, IMO, pp. 1-16.

Miles, C.O., Samdal, I.A., Aasen, J.A.G., Jensen, D.J., Quilliam, M.A., Petersen, D., Briggs, L.M., Wilkins, A.L., Rise, F., Cooney, J.M., MacKenzie, A.L., 2005a. Evidence for numerous analogs of yessotoxin in Protoceratium reticulatum. Harmful Algae 4 (6), 1075-1091.

Miles, C.O., Wilkins, A.L., Hawkes, A.D., Selwood, A.I., Jensen, D.J., Munday, R., Cooney, J.M., Beuzenberg, V., 2005b. Polyhydroxylated amide analogs of yessotoxin from Protoceratium reticulatum. Toxicon 45 (1), 61-71.

Moita, M.T., Sampayo, M.A. de M., 1993. Are there cysts in the genus Dinophysis? In: Srnayda, T.J., Shimizu, Y. (Eds.), Toxic Phytoplankton Blooms in the Sea. Proc 5th Int Conference on Toxic Marine Phytoplankton, 28 Oct-1 Nov 1991. New-port, USA. Elsevier Sci Pub, New York, pp. 153-157.

Montresor, M., Zingone, A., 1988. Scrippsiella precaria sp. nov. (Dinophyceae), a marine dinoflagellate from the Gulf of Naples. Phycologia 27, 387-394.

Montresor, M., Zingone, A., Marino, D., 1993. The calcareous resting cyst of Pentapharsodinium tyrrhenicum comb. nov. (Dinophyceae). J. Phycol. 29 (2), 223-230.

Montresor, M., Montesarchio, E., Zingone, A., Marino, D., 1994. Calcareous dinoflagellate cysts in marine sediments of the Gulf of Naples (Mediterranean Sea). Rev. Palaeobot. Palynol. 84, 45-56.

Montresor, M., Janofske, D., Willems, H., 1997. The cyst-theca relationship in Calciodinellum operosum emend. (Peridiniales, Dinophyceae) and a new approach for the study of calcareous cysts. J. Phycol. 33, 122-131.

Montresor, M., Zingone, A., Sarno, D., 1998. Dinoflagellate cyst production at a coastal Mediterranean site. J. Plankton Res. 20, 2291-2312.

Montresor, M., Sgrosso, S., Procaccini, G., Kooistra, W.H.C.F., 2003. Intraspecific diversity in Scrippsiella trochoidea (Dinophyceae): evidence for cryptic species Phycologia 42 (1), 56-70.

Montresor, M., John, U., Beran, A., Medlin, L.K., 2004. Alexandrium tamutum sp. nov. (Dinophyceae): a new nontoxic species in the genus Alexandrium. J. Phycol. 40, 398-411.

Morzadec-Kerfourn, M.-T., 1977. Les cystes de dinoflagellés dans les sédiments récents le long des côtes Bretonnes. Rev. Micropaleontol. 20, 157-166.

Mozetič, P., Cangini, M., Francé, J., Bastianini, M., Bernardi Aubry, F., Bužančić, M., Cabrini, M., Cerino, F., Čalić, M., D'Adamo, R., Drakulović, D., Finotto, S., Fornasaro, D., Grilli, F., Kraus, R., Kužat, N., Marić Pfannkuchen, D., Ninčević Gladan, Ž., Pompei, M., Rotter, A., Servadei, I., Skejić, S., 2019. Phytoplankton diversity in Adriatic ports: lessons from the port baseline survey for the management of harmful algal species. Mar. Pollut. Bull (This issue. In press).

Murphy, K.R., Ritz, D., Hewitt, C.L., 2002. Heterogeneous zoo-plankton distribution in a ship's ballast tanks. J. Plankton Res. 24 (7), 729-734.

Nagai, S., Matsuyama, Y., Takayama, H., Kotani, Y., 2002. Morphology of Polykrikos 
kofoidii and P. schwartzii (Dinophyceae, Polykrikaceae) cysts obtained in culture. Phycologia 41, 319-327.

Nguyen-Ngoc, L., 2004. An autecological study of the potentially toxic dinoflagellate Alexandrium affine isolated from Vietnamese waters. Harmful Algae 3 (2), 117-129. https://doi.org/10.1016/51568-9883 (03)00062-3.

Nichetto, P., Honsell, G., Bressan, G., 1995. First survey of dinoflagellate cysts in the Gulf of Trieste (Northern Adriatic Sea). In: Lassus, P., Arzul, G., Erard-Le Denn, E., Gentien, P., Marcaillou-Le Baut, C. (Eds.), Harmful Marine Algal Blooms. Lavoisier Pub, Paris, pp. 205-211.

Ninčević Gladan, Ž., Magaletti, E., Scarpato, A., Azzurro, E., Bacci, T., Berto, D., Dulčić, J., Gennaro, P., Marusso, V., Penna, M., Rampazzo, F., Rende, F.S., Romanelli, G., Vidjak, O., Trabucco, B., Žuljević, A., 2014. BALMAS Port Baseline Survey Protocol. Protocol. BALMAS Project. Work Package 5.1. (28 pp).

Olenin, S., Gollasch, S., Jonusas, S., Rimkute, I., 2000. En-route investigation of plankton in ballast water on ship's voyage from the Baltic Sea to the open Atlantic coast of Europe. Int. Rev. Hydrobiol. 85, 577-596.

Orlić, M., Kuzmić, M., Pasarić, Z., 1994. Response of the Adriatic Sea to the bora and sirocco forcing. Cont. Shelf Res. 14 (1), 91-116.

Paz, B., Riobo, P., Fernandez, A.L., Fraga, S., Franco, J.M., 2004. Production and release of yessotoxins by the dinoflagellates Protoceratium reticulatum and Lingulodinium polyedrum in culture. Toxicon 44, 251-258.

Paz, B., Vázquez, J.A., Riobó, P., Franco, J.M., 2006. Study of the effect of temperature, irradiance and salinity on growth and yessotoxin production by the dinoflagellate Protoceratium reticulatum in culture by using a kinetic and factorial approach. Mar. Environ. Res. 62, 286-300.

Penna, A., Bertozzini, E., Battocchi, C., Galluzzi, L., Giacobbe, M.G., Vila, M., Garces, E., Lugliè, A., Magnani, M., 2007. Monitoring of HAB species in the Mediterranean Sea through molecular methods. J. Plankton Res. 29 (1), 19-38. https://doi.org/10. 1093/plankt/fbl053.

Penna, A., Fraga, S., Masó, M., Giacobbe, M.G., Bravo, I., Garcés, E., Vila, M., Bertozzini, E., Andreoni, F., Lugliè, A., Vernesi, C., 2008. Phylogenetic relationships among the Mediterranean Alexandrium (Dinophyceae) species based on sequences of 5.8S gene and Internal Transcript Spacers of the rRNA operon. Eur. J. Phycol. 43 (2), 163-178.

Perini, F., Bastianini, M., Capellacci, S., Pugliese, L., DiPoi, E., Cabrini, M., Buratti, S., Marini, M., Penna, A., 2019. Molecular Methods for Cost-efficient Monitoring of HAB (Harmful Algal Bloom) Dinoflagellate Resting Cysts. (This issue. In press).

Persson, A., Godhe, A., Karlson, B., 2000. Dinoflagellate cysts in recent sediments from the west coast of Sweden. Bot. Mar. 43, 69-79.

Pfiester, L.A., Anderson, D.M., 1987. Dinoflagellate reproduction. In: Taylor, F.J.R (Ed.), The Biology of Dinoflagellates. Botanical Monographs. 21. Blackwell ScientificPublications, Oxford, pp. 611-648.

Pitcher, G.C., Joyce, L.B., 2009. Dinoflagellate cyst production on the southern Namaqua shelf of the Benguela upwelling system. J. Plankton Res. 31 (8), 865-875.

Pospelova, V., de Vernal, A., Pedersen, T.F., 2008. Distribution of dinoflagellate cysts in surface sediments from the northeastern Pacific Ocean $\left(43-25^{\circ} \mathrm{N}\right)$ in relation to seasurface temperature, salinity, productivity and coastal upwelling. Mar. Micropaleontol. 68, 21-48.

Rhodes, L., McNabb, P., de Salas, M., Briggs, L., Beuzenberg, V., Gladstone, M., 2006. Yessotoxin production by Gonyaulax spinifera. Harmful Algae 5 (2), 148-155.

Riccardi, M., Guerrini, F., Roncarati, F., Milandri, A., Cangini, M., Pigozzi, S., Riccardi, E., Ceredi, A., Ciminiello, P., Dell'Aversano, C., Fattorusso, E., Forino, M., Tartaglione, L., Pistocchi, R., 2009. Gonyaulax spinifera from the Adriatic Sea: toxin production and phylogenetic analysis. Harmful Algae 8, 279-290.

Rigby, G.R., Hallegraeff, G.M., 1994. The transfer and control of harmful marine organisms in shipping ballast water: behavior of marine plankton and ballast water exchange trials on the MV "Iron Whyalla". J. Mar. Environ. Eng. 1, 91-110.

Rigby, G.R., Hallegraeff, G.M., 1996. Ballast water controls to minimize the translocation and establishment of toxic phytoplankton: what progress have we made and where are we going? In: Seventh International Conference on Toxic Phytoplankton, Sendai Japan, pp. 201-204.

Rochon, A., de Vernal, A., Turon, J.L., Matthiessen, J., Head, M.J., 1999. Distribution of recent dinoflagellate cysts in surface sediments from the North Atlantic Ocean and adjacent seas in relation to sea-surface parameters. In: AASP Contribution Series 35 American Association of Stratigraphic Palynologists Foundation (152 pp).

Rogelja, M., Cibic, T., Rubino, F., Belmonte, M., Del Negro, P., 2018. Active and resting microbenthos in differently contaminated marine coastal areas: insights from the Gulf of Trieste (northern Adriatic, Mediterranean Sea). Hydrobiologia 806, 283-301. https://doi.org/10.1007/s10750-017-3366-1.

Rubino, F., Belmonte, G., Miglietta, A.M., Geraci, S., Boero, F., 2000. Resting stages of plankton in recent North Adriatic sediments. Mar. Ecol. 21 (34), 263-284.

Rubino, F., Moscatello, S., Saracino, O.D., Fanelli, G., Belmonte, G., Boero, F., 2002. Plankton-derived resting stages in marine coastal sediments along the Salenta Peninsula (Apulia, South-Eastern Italy). Mar. Ecol. 23, 329-339.

Rubino, F., Saracino, O.D., Moscatello, S., Belmonte, G., 2009. An integrated water/sediment approach to study plankton (a case study in the southern Adriatic Sea). J. Mar. Syst. 78 (4), 536-546.

Rubino, F., Belmonte, M., Caroppo, C., Giacobbe, M., 2010. Dinoflagellate cysts from surface sediments of Syracuse Bay (Western Ionian Sea, Mediterranean). Deep-Sea Res. II 57 (3), 243-247.

Rubino, F., Belmonte, M., Galil, B.S., 2017. Plankton resting stages in recent sediments of Haifa port, Israel (Eastern Mediterranean) - distribution, viability and potential environmental consequences. Mar. Pollut. Bull. 116 (1-2), 258-269. https://doi.org/ 10.1016/j.marpolbul.2016.12.078.

Ruiz, G.M., Rawlings, T.K., Dobbs, F.C., Drake, L.A., Mullady, T., Huq, A., Colwell, R.R., 2000. Global spread of microorganisms by ships-ballast water discharged from vessels harbours a cocktail of potential pathogens. Nature 408, 49-50.
Samdal, I.A., Naustvoll, L.J., Olseng, C.D., Briggs, L.R., Miles, C.O., 2004. Use of ELISA to identify Protoceratium reticulatum as a source of yessotoxin in Norway. Toxicon 44 (1), 75-82.

Sangiorgi, F., Donders, T.H., 2004. Reconstructing 150 years of eutrophication in the north-western Adriatic. Sea (Italy) using dinoflagellate cysts, pollen and spores. Estuar. Coast. Shelf Sci. 60 (1), 69-79.

Saracino, O.D., Rubino, F., 2006a. Ballast water monitoring in cargo vessels in Taranto harbour (Ionian Sea). Biol. Mar. Mediterr. 13 (1), 1052-1054.

Saracino, O.D., Rubino, F., 2006b. Phytoplankton composition and distribution along the Albanian coast, South Adriatic Sea. Nova Hedwigia 83 (1-2), 253-266.

Satta, C.T., Anglès, S., Garcés, E., Lugliè, A., Padedda, B.M., Sechi, N., 2010. Dinoflagellate cysts in recent sediments from two semienclosed areas of the Western Mediterranean Sea subject to high human impact. Deep-Sea Res. II 57, 256-267.

Satta, C.T., Anglès, S., Lugliè, A., Guillén, J., Sechi, N., Camp, J., Garcés, E., 2013. Studies on dinoflagellate cyst assemblages in two estuarine Mediterranean bays: a useful tool for the discovery and mapping of harmful algal species. Harmful Algae 24, 65-79.

Satta, C.T., Anglès, S., Garcés, E., Sechi, N., Pulina, S., Padedda, B.M., Stacca, D., Lugliè, A., 2014. Dinoflagellate cyst assemblages in surface sediments from three shallow Mediterranean lagoons (Sardinia, North Western Mediterranean Sea). Estuar. Coasts 37, 646-663. https://doi.org/10.1007/s12237-013-9705-1.

Shin, H.H., Baek, S.H., Li, Z., Han, M.-S., Oh, S.J., Youn, S.-H., Kim, Y.S., Kim, D., Lim, W.A., 2014. Resting cysts, and effects of temperature and salinity on the growth of vegetative cells of the potentially harmful species Alexandrium insuetum Balech (Dinophyceae). Harmful Algae 39, 175-184. https://doi.org/10.1016/j.hol.2014.07. 012.

Spatharis, S., Danielidis, D., Tsirtsis, G., 2007. Recurrent Pseudo-nitzschia calliantha (Bacillariophyceae) and A. insuetum (Dinophyceae) winter blooms induced by agricultural run-off. Harmful Algae 6, 811-822.

Supić, N., Orlić, M., Degobbis, D., 2003. Istrian coastal countercurrent in the year 1997. Il Nuovo Cimento 26, 117-131.

Supić, N., Kraus, R., Kuzmić, M., Paschini, E., Precali, R., Russo, A., Vilibić, I., 2012. Predictability of northern Adriatic winter conditions. J. Mar. Syst. 90 (1), 42-57.

Taylor, F.J.R., 1987. The Biology of Dinoflagellates. Blackwell University of California Press, USA (798 pp).

Thorsen, T.A., Dale, B., 1997. Dinoflagellate cysts as indicators of pollution and past climate in a Norwegian fjord. The Holocene $7,433-446$.

Toebe, K., Alpermann, T.J., Tillmann, U., Krock, B., Cembella, A., John, U., 2013. Molecular discrimination of toxic and non-toxic Alexandrium species (Dinophyta) in natural phytoplankton assemblages from the Scottish coast of the North Sea. Eur. J. Phycol. 48 (1), 12-26.

Tolomio, C., Bullo, L., 2001. Prelievi giornalieri di fitoplancton in una stazione del bacino meridionale della Laguna di Venezia; Aprile 1993-Marzo 1994. Boll. Mus. Civ. Stor. Nat. Venezia 52, 3-23.

Totti, C., Civitarese, G., Acri, F., Barletta, D., Candelari, G., Paschini, E., Solazzi, A., 2000. Seasonal variability of phytoplankton populations in the middle Adriatic sub-basin. J. Plankton Res. 22 (9), 1735-1756.

Triki, H.Z., Laabir, M., Lafabrie, C., Malouche, D., Bancon-Montigny, C., Gonzalez, C., Daly-Yahia, O.K., 2017. Do the levels of industrial pollutants influence the distribution and abundance of dinoflagellate cysts in the recently-deposited sediment of a Mediterranean coastal ecosystem? Sci. Total Environ. 595, 380-392.

Umgiesser, U., Sclavo, M., Carniel, S., Bergamasco, A., 2004. Exploring the bottom stress variability in the Venice Lagoon. J. Mar. Syst. 51, 161-178. https://doi.org/10.1016/ j.jmarsys.2004.05.023.

Utermöhl, H., 1958. Zur Vervollkommnung der quantitativen Phytoplankton-Methodik. Mitt. Int. Ver. Theor. Angew. Limnol. 9, 1-38.

Viličić, D., Marasović, I., Mioković, D., 2002. Checklist of phytoplankton in the eastern Adriatic Sea. Acta Bot. Croat. 61 (1), 57-91.

Viličić, D., Djakovac, T., Burić, Z., Bosak, S., 2009. Composition and annual cycle of phytoplankton assemblages in the northeastern Adriatic Sea. Bot. Mar. 52 (4), 291-305.

Villac, M.C., Persich, G., Fernandes, L., Paranhos, R., Dias, C., Bonecker, S., Garcia, V., Odebrecht, C., Tenenbaum, D., Tristão, M.L., Andrade, S., Fadel, A., 2001. Ballast water exchange: testing the dilution method (Petrobrás, Brazil). In: Hallegraeff, G.M., Blackburn, S.I., Bolch, C.J., Lewis, R.J. (Eds.), Harmful Algal Blooms 2000. International Oceanographic Commission, Paris, pp. 470-473.

Walker, L.M., 1984. Life histories, dispersal and surival in marine, planktonic dinoflagellates. In: Steidinger, K.A., Walker, L.M. (Eds.), Marine Plankton Life Cycle Strategies. CRC Press, Florida, pp. 19-35.

Wall, D., Dale, B., Lohman, G.P., Smith, W.K., 1977. The environmental and climatic distribution of dinoflagellate cysts in modern marine sediments from regions in the north and south Atlantic oceans and adjacent seas. Mar. Micropaleontol. 2, 121-200.

Wang, Z.H., Qi, Y.Z., Yang, Y.F., 2007. Cyst formation: an important mechanism for the termination of Scrippsiella trochoidea (Dinophyceae) bloom. J. Plankton Res. 29 (2), 209-218.

Williams, R.J., Griffiths, F.B., Van der Wal, E.J., Kelly, J., 1988. Cargo vessel ballast water as a vector for the transport of non-indigenous marine species. Estuar. Coast. Shelf Sci. 26, 409-420.

Williamson, M., 1996. Biological Invasions. Vol. 15 Springer Science and Business Media (244 pp).

Wonham, M.J., Walton, W.C., Ruiz, G.M., Frese, A.M., Galil, B.S., 2001. Going to the source: role of the invasion pathway in determining potential invaders. Mar. Ecol. Prog. Ser. 215, 1-12.

Yasumoto, T., Satake, M., 1998. New toxins and their toxicological evaluations. In: Reguera, B., Blanco, J., Fernandez, L.L., Wyatt, T. (Eds.), Harmful Algae. Xunta de Galicia and Intergovernmental Oceanographic Commission of UNESCO, pp. 461-464.

Zingone, A., Totti, C., Sarno, D., Cabrini, M., Caroppo, C., Giacobbe, M.G., Luglie, A., 
Nuccio, C., Socal, G., 2010. Fitoplancton: metodiche di analisi quali-quantitativa. In: Socal, G., Buttino, I., Cabrini, M., Mangoni, O., Penna, A., Totti, C. (Eds.),

Metodologie di studio del plancton marino. Manuali e Linee Guida. ISPRA-SIBM, Roma, pp. 213-237.

Zonneveld, K.A.F., Susek, E., Fischer, G., 2010. Seasonal variability of the organic-walled dinoflagellate cyst production in the coastal upwelling region off Cape Blanc (Mauritania): a five-year survey. J. Phycol. 46, 202-215.

Zonneveld, K.A.F., Marret, F., Versteegh, G.J.M., Bogus, K., Bonnet, S., Bouimetarhan, I.,
Crouch, E., Vernal, A., Elshanawany, R., Edwards, L., Esper, O., Forke, S., Grøsfjeld, K., Henry, M., Holzwarth, U., Kielt, J.-F., Kim, S.-Y., Ladouceur, S., Ledu, D., Chen, L., Limoges, A., Londeix, L., Lu, S.-H., Mahmoud, M.S., Marino, G., Matsouka, K.,

Matthiessen, J., Mildenhal, D.C., Mudie, P., Neil, H.L., Pospelova, V., Qi, Y., Radi, T., Richerol, T., Rochon, A., Sangiorgi, F., Solignac, S., Turon, J.-L., Verleye, T., Wang,

Y., Wang, Z., Young, M., 2013. Atlas of modern dinoflagellate cyst distribution based on 2405 data points. Rev. Palaeobot. Palynol. 191, 1-197. https://doi.org/10.1016/j. revpalbo.2012.08.003. 